\title{
A Cnoidal Approximation Wave Theory
}

\author{
Rodney J. Sobey \\ Department of Civil and Environmental Engineering, \\ Imperial College London, \\ London SW7 2AZ, UK \\ E-mail: r.j.sobey@imperial.ac.uk
}

\section{Engineering and Computational Mechanics, 156, 201-216, 2012}

25 June 2012

\begin{abstract}
Periodic progressive waves in shallow to transitional water are described by a Cnoidal Approximation wave theory. The formulation is analytical and largely classical. Periodic waves are assumed, so that the theory does not extend to solitary waves. The solution is completed numerically, as a problem in nonlinear optimisation. The suitability of the theory in shallow to transitional water is demonstrated to $88 \%$ of the limit wave height.
\end{abstract}

Keywords Cnoidal waves, extreme wave kinematics, limit waves, nonlinear optimisation, numerical, progressive waves, short waves, steady wave theory, Stokes waves.

\section{Introduction}

The extreme wave credibility of analytical shallow water (cnoidal) wave theory to fifth order extends only to about $50 \%$ of the limit wave height in very shallow water, rising to about $70 \%$ in transitional water. Increasing the order of approximation is expected to result in improvements in the predictive credibility. The analytical expressions for the coefficients are already very long at fifth order, and a higher order analytical theory will further increase the opportunity for transcription errors that have so compromised analytical theories in the literature. This is not a pragmatic approach.

For deep water (Stokes) waves, analytical theories to fifth order have credibility much closer to the limit wave. This credibility has been further enhanced by a hybrid analyticalnumerical extension of the Stokes approximation where the analytical formulation follows 


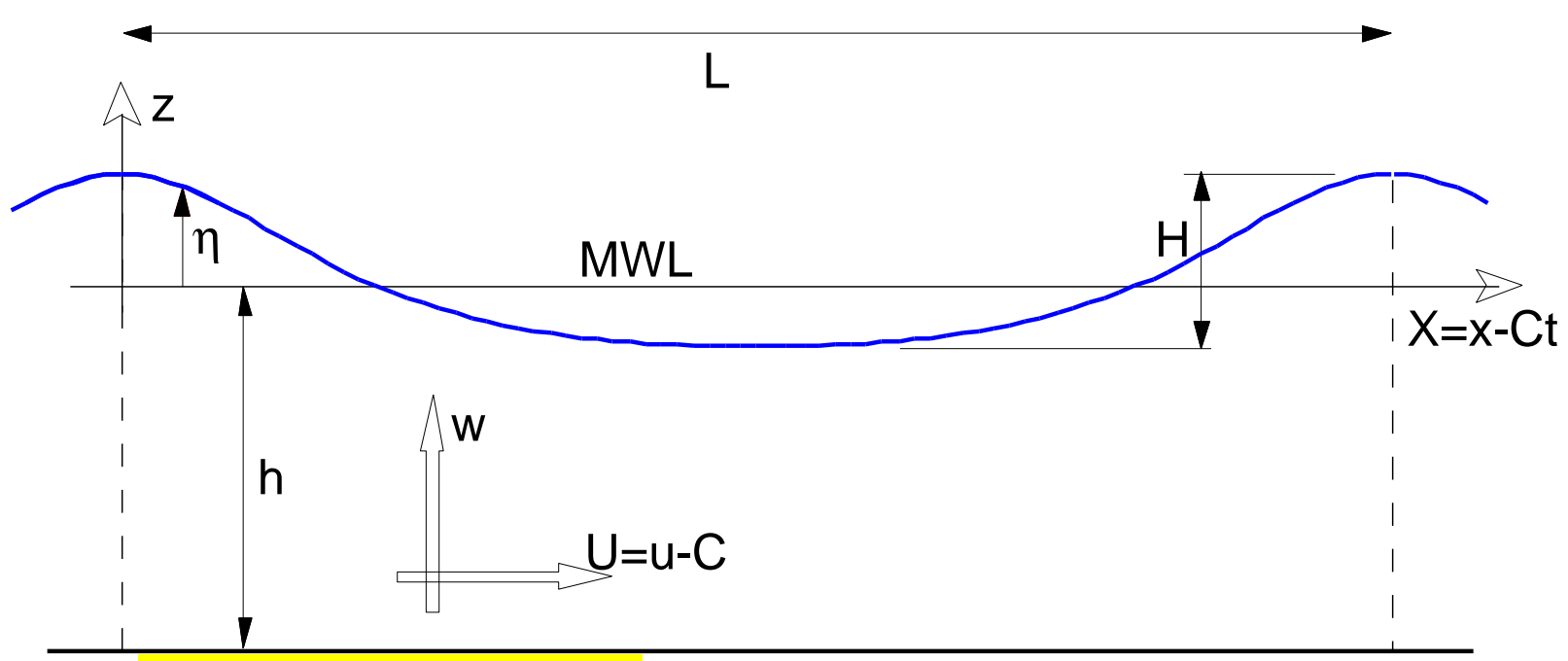

Figure 1: Definition sketch for steady progressive wave.

the Stokes approach with the unknowns of the theory assigned numerically rather than analytically. Such a Stokes approximation has alternatively been called stream function wave theory or Fourier approximation wave theory. These Stokes approximation theories have had considerable success. By default almost, their domain of applicability has been pushed into the shallow water region through very significant increases in the order of approximation.

A cnoidal approximation theory is here introduced. It is a hybrid analytical-numerical extension of the cnoidal theory where the analytical formulation follows the cnoidal approach with the unknowns of the theory assigned numerically rather than analytically. There are a number of obvious parallels with the Stokes approximation approach, but equally a number of issues unique to the shallow water context. The theory is formulated and demonstrated below. Its credibility at very moderate order extends to about $88 \%$ of the limit wave.

\section{Mathematical Formulation of Steady Wave Theory}

Progressive waves of permanent form are steady in a frame of reference moving with the crest at the phase speed $C$. Accordingly, it is convenient to adopt a moving $(X=x-C t, z)$ reference frame (see Figure 1) that is located at the mean water level (MWL) and that moves at speed $C$ with the wave crest. In this $(X, z)$ frame, the flow is steady, whereas in the fixed $(x, z, t)$ reference frame, the flow is unsteady. Periodic waves and incompressible and irrotational flow are assumed.

The mathematical formulation may be presented in terms of the Euler equations, the velocity potential function, the stream function or the complex potential function. The choice of the stream function $\Psi(X, z)$ in the steady frame has some minor advantage in 
the representation of the kinematic free surface boundary condition (as it does not require time or spatial derivatives of the primitive variables $\eta$ and $\Psi$ ).

In terms of the stream function, the field equation representing mass conservation and irrotational flow is the Laplace equation

$$
\frac{\partial^{2} \Psi}{\partial X^{2}}+\frac{\partial^{2} \Psi}{\partial z^{2}}=0
$$

where the velocity components $(U=u-C, w)$ are $(\partial \Psi / \partial z,-\partial \Psi / \partial X)$.

Momentum conservation throughout the fluid is represented by the irrotational Bernoulli equation in the steady frame

$$
\frac{1}{2}\left(U^{2}+w^{2}\right)+\frac{p}{\rho}+g z=R
$$

where $R$ is the Bernoulli constant in the steady frame.

In terms of the stream function, the complete boundary constraints are as follows.

(1) The bottom boundary condition (BBC), representing no flow through the horizontal bed, is

$$
\Psi(X,-h)=0 \quad \text { or } \quad \Psi(X, z)=0 \quad \text { at } \quad z=-h
$$

(2) The kinematic free-surface boundary condition (KFSBC), representing no flow through the free surface at $\eta(X)$, is

$$
\Psi(X, \eta(X))=-Q \quad \text { or } \quad \Psi(X, z)=-Q \quad \text { at } \quad z=\eta(X)
$$

where $-Q$ is the constant volume flow rate per unit width under the steady wave. $Q$ is numerically positive and this flow is in the negative $X$ direction. The zero datum for the stream function was established by the BBC.

(3) The dynamic free surface boundary condition (DFSBC), representing constant atmospheric pressure on the free surface, is

$$
\frac{1}{2}\left(U^{2}+w^{2}\right)+g \eta=R \quad \text { at } \quad z=\eta(X)
$$

which follows directly from the irrotational Bernoulli equation in the steady frame.

(4) The wave is periodic. The periodic lateral boundary conditions (PLBC) are

$$
\Psi(X+L, z)=\Psi(X, z), \quad \eta(X+L)=\eta(X)
$$

where $L$ is the wave length. Solitary waves with infinite $L$ are excluded.

(5) The wave height is defined as

$$
H=\eta(0)-\eta(L / 2)
$$


(6) Mass conservation requires an invariant mean water level (MWL) such that

$$
\int_{0}^{L / 2} \eta(X) d X=0
$$

In principle, the unknowns of a steady wave solution will be the stream function $\Psi(X, z)$, the water surface profile $\eta(X)$, the wave length $L$, the current or mean fluid speed $-\bar{U}$ at any elevation that is always submerged below the trough, the volume flux $-Q$ and the Bernoulli constant $R$. In specific nonlinear wave theories, the stream function, the water surface profile and the wavelength are represented in terms of other parameters, which take their place as unknowns in the theory.

The given parameters defining a steady wave solution are generally the wave height $H$, the water depth $h$, the wave period $T(=2 \pi / \omega)$ and either the co-flowing Eulerian current $U_{E}$ or the wave-averaged mass transport velocity or Stokes drift $U_{S}$.

\section{Dispersion Relationship}

Steady wave theory seeks to predict the flow in the moving and steady flow reference frame. In this frame, it will predict both the current $-\bar{U}$ and the volume flow rate $-Q$ under the steady wave, respectively

$$
-\bar{U}=\frac{1}{L} \int_{0}^{L} U(X, z) d X, \quad-Q=\int_{-h}^{\eta(X)} U(X, z) d z
$$

In the $\bar{U}$ definition, the elevation $z$ can be any elevation at or below the trough; $z=-h$ is often convenient. In the $Q$ definition, the location $X$ can be any location, as the flow is steady.

Steady wave theory does not predict the actual speed $C$ of this frame. $C$ is measured in the fixed and unsteady frame, and is dependent also on background flow conditions.

The current in the steady frame is $-\bar{U}$ and the steady frame is moving at speed $C$, so that the current in the fixed and unsteady frame is $U_{E}=-\bar{U}+C$. Similarly, the depthaveraged mass transport velocity in the steady frame is $-Q / h$, so that the depth-averaged mass transport velocity in the fixed and unsteady frame is $U_{S}=-Q / h+C$.

Transformation between the frames requires knowledge of both the current and the wave period.

(i) Where the Eulerian current $U_{E}$ is known, the phase speed is

$$
C=\bar{U}+U_{E}
$$

The depth-averaged mass transport velocity or Stokes drift $U_{S}$ is then available from

$$
C=\frac{Q}{h}+U_{S}
$$


(ii) Where the depth-averaged mass transport velocity $U_{S}$ is known, Eq. 11 predicts the phase speed. Eq. 10 then becomes the definition equation for the Eulerian current.

These alternative coordinate transformations are often called Stokes' first and second "definitions" of phase speed.

In either case, the space and time periodicity of the progressive wave of permanent form requires

$$
C=\frac{L}{T}
$$

as a simultaneous equation. The wave period $T$ is the (absolute) period measured in a fixed reference frame, e.g., by a wave staff or buoy at a fixed position. Similarly, $U_{E}$ is the time-mean Eulerian current in the fixed reference frame, as measured by a current meter deployed at a fixed position below the wave trough.

Taken together,

(i) Eqs. 10 and 12 where the Eulerian current is known -

$$
\frac{L}{T}=\bar{U}+U_{E}
$$

(ii) or Eqs. 11 and 12 where the depth-averaged mass transport velocity is known -

$$
\frac{L}{T}=\frac{Q}{h}+U_{S}
$$

relate the space and time periodicity. This is the dispersion relationship for the progressive wave. Whichever equation is not used becomes the definition equation for the other "current." With $L, \bar{U}$ and $Q$ predicted by a steady wave theory, the details will vary with the wave theory.

\section{Kinematics}

Following (Lamb 1932, p.423-4) and a number of subsequent analytical theories (Keller 1948, Laitone 1960, Fenton 1979, Sobey 2012), a suitable shallow-water focussed solution of the field Laplace Eq. 1 is the truncated series

$$
\Psi(X, z)=\left(g h^{3}\right)^{1 / 2} \sum_{k=0}^{N} \Psi_{k}(\chi, \zeta), \quad \Psi_{k}(\chi, \zeta)=(-1)^{k} \frac{(1+\zeta)^{2 k+1}}{(2 k+1) !} f_{k}(\chi)
$$

in which

$$
f_{0}(\chi)=f(\chi) \text { and } f_{k}(\chi)=\alpha^{2} \frac{d^{2}}{d \chi^{2}} f_{k-1}, k=1, \ldots
$$

and $N$ is the truncation order. The dimensionless independent variables are

$$
\chi=\alpha \frac{X}{h}, \quad \zeta=\frac{z}{h}
$$


where

$$
\alpha=\frac{2 \mathrm{~K}(m) h}{L}
$$

in which $\mathrm{K}(m)$ is the Jacobian elliptic integral of the first kind with parameter $m$. The shallow-water focus is explicit in the $\alpha$ stretching of the horizontal independent variable in Eq. 17. Note that Eq. 15 exactly satisfies the bottom boundary condition (Eq. 3).

The field equation (Eq. 1) becomes, non-dimensionally,

$$
\begin{aligned}
\alpha^{2} \frac{\partial^{2} \Psi}{\partial \chi^{2}}+\frac{\partial^{2} \Psi}{\partial \zeta^{2}}= & \alpha^{2} \frac{\partial^{2} \Psi_{0}}{\partial \chi^{2}}+\frac{\partial^{2} \Psi_{0}}{\partial \zeta^{2}}+\alpha^{2} \frac{\partial^{2} \Psi_{1}}{\partial \chi^{2}}+\frac{\partial^{2} \Psi_{1}}{\partial \zeta^{2}} \\
& +\ldots+\alpha^{2} \frac{\partial^{2} \Psi_{k}}{\partial \chi^{2}}+\frac{\partial^{2} \Psi_{k}}{\partial \zeta^{2}}+\ldots+\alpha^{2} \frac{\partial^{2} \Psi_{N}}{\partial \chi^{2}}+\frac{\partial^{2} \Psi_{N}}{\partial \zeta^{2}}
\end{aligned}
$$

It follows from Eqs. 15 and 16 that $\partial^{2} \Psi_{0} / \partial \zeta^{2}=0$ and

$$
\alpha^{2} \frac{\partial^{2} \Psi_{k-1}}{\partial \chi^{2}}+\frac{\partial^{2} \Psi_{k}}{\partial \zeta^{2}} \equiv 0, \quad k=1,2, \ldots N
$$

identifying a Laplace field residual, $\alpha^{2} \partial^{2} \Psi_{N} / \partial \chi^{2}$, at truncation.

In a classical analytical small parameter expansion theory, strict truncation of all terms in the formulation at order $N$ conveniently results in a vanishing Laplace field residual. A numerical theory imposes neither strict truncation at order $N$ nor the expectation that contributions at progressively higher orders are routinely smaller in magnitude. A significant Laplace field residual is possible. This must be acknowledged in the problem formulation.

In addition to the boundary constraints (1) through (6) (Eqs. 3 through 8), there must be added

(7) The free surface Laplace condition

$$
\frac{\partial^{2} \Psi}{\partial X^{2}}+\frac{\partial^{2} \Psi}{\partial z^{2}}=\frac{\partial U}{\partial z}-\frac{\partial w}{\partial X}=0 \quad \text { at } \quad z=\eta(X)
$$

Because of the $(1+\zeta)$ multiplier in the stream function (Eq. 15), the Laplace field residual is zero at the bed $z=-h$, and largest at the water surface $z=\eta(X)$. Eq. 21 imposes a field constraint on the Laplace residual.

In any steady wave theory, the most vulnerable aspect of the predicted kinematics is routinely the acceleration components $D u / D t$ and $D w / D t$

$$
\frac{D u}{D t}=U \frac{\partial U}{\partial X}+w \frac{\partial U}{\partial z}, \quad \frac{D w}{D t}=U \frac{\partial w}{\partial X}+w \frac{\partial w}{\partial z}
$$

The vulnerability stems from the quadratic terms on the right hand side together with the dependence on spatial derivatives of the velocity components, which are in turn spatial second derivatives of the stream function. These spatial derivatives are related through the mass conservation equation

$$
\frac{\partial U}{\partial X}+\frac{\partial w}{\partial z}=0
$$


and the irrotational flow constraint

$$
\frac{\partial U}{\partial z}-\frac{\partial w}{\partial X}=0
$$

Eq. 23 is exactly satisfied by the adoption of a stream function. Eq. 24 becomes the Laplace field equation, as indicated in Eq. 21. A classical steady wave formulation does not include the velocity gradients. As suggested by Eq. 19 and the associated discussion, the inclusion of the irrotational flow constraint in the problem formulation (Eq. 21) may benefit the predictive precision of the acceleration components for shallow water waves.

Following a number of analytical theories (Keller 1948, Laitone 1960, Fenton 1979, Sobey 2012), the function $f(\chi)$ in the stream function is represented as the truncated series

$$
f(\chi)=\sum_{j=0}^{N} A_{j} \mathrm{cn}^{2 j}(\chi ; m)
$$

and the water surface elevation as the truncated series

$$
\eta(\chi)=\sum_{j=0}^{N} B_{j} \mathrm{cn}^{2 j}(\chi ; m), \quad \eta(X)=h \eta(\chi)
$$

in which $\mathrm{cn}(\chi ; m)$ is the Jacobian elliptic cosine function. Note that the Eq. 18 definition for $\alpha$ results in Eqs. 25 and 26 exactly satisfying the periodic lateral boundary conditions (Eq. 6).

There are peripheral suggestions in the solitary wave literature (e.g. Engevik 2003) that infinite shallow water series solutions are divergent. Relevance to the present context is very tenuous. Firstly, solitary waves are specifically excluded as they are not periodic waves (Equations 6, 13, 14); solutions very close to $m=1$ are sought, but not at $m=1$ where $\mathrm{K}(m), \alpha$ and $L$ are infinite. Secondly, the adopted series (Equations 15, 25, 26) are not infinite but truncated at $N$. Minimising the truncation error (Equation 21) is explicit in the problem formulation.

The $f_{k}(\chi)$ in Eq. 15 require recurring second derivatives, which are represented as

$$
\begin{aligned}
& f_{k}(\chi)=\sum_{j=0}^{2 N+1} A_{j}^{k} \operatorname{cn}^{2 j}(\chi ; m) \\
& \text { in which }\left[A_{j}^{0}\right]=\left[A_{j}\right] \text { and }\left[A_{j}^{k}\right]=\alpha^{2}\left[A_{j}^{k-1}\right] \mathbb{F} \text { for } k=1 \ldots N
\end{aligned}
$$

where $\left[A_{j}^{k}\right]$ are $1 \times(2 N+2)$ coefficient vectors and $\mathbb{F}$ is a $(2 N+2) \times(2 N+2)$ transformation matrix (see Appendix A) that provides the second derivative of $\mathrm{cn}^{2 j}(\chi ; m)$. As a consequence of the analytical expression for $d^{2} \mathrm{cn}^{2 j}(\chi) / d \chi^{2}$ (Eq. 56), each increment in $k$ expands the row width of $\left[A_{j}^{k}\right]$ by one element. The first $(k=0)$ row has active entries in columns 1 through $N+1$ and $N+1$ trailing zeros, the second $(k=1)$ row has active entries in columns 1 through $N+2$ and $N$ trailing zeros, ... and finally the row $k=N+1$ has active entries in columns 1 through $2 N+2$ and no trailing zeros. 
The dynamic free surface boundary condition requires also the velocity components

$$
\begin{aligned}
& U(X, z)=\frac{\partial \Psi(X, z)}{\partial z}=\frac{1}{h} \sum_{k=0}^{N}(-1)^{k} \frac{(1+\zeta)^{2 k}}{(2 k) !} f_{k}(\chi) \\
& w(X, z)=-\frac{\partial \Psi(X, z)}{\partial X}=-\frac{\alpha}{h} \sum_{k=0}^{N}(-1)^{k} \frac{(1+\zeta)^{2 k+1}}{(2 k+1) !} g_{k}(\chi)
\end{aligned}
$$

and the free surface Laplace condition the velocity gradients

$$
\begin{aligned}
\frac{\partial U}{\partial z} & =\frac{1}{h^{2}} \sum_{k=1}^{N}(-1)^{k} \frac{(1+\zeta)^{2 k-1}}{(2 k-1) !} f_{k}(\chi) \\
\frac{\partial w}{\partial X} & =-\frac{\alpha^{2}}{h^{2}} \sum_{k=0}^{N}(-1)^{k} \frac{(1+\zeta)^{2 k+1}}{(2 k+1) !} \mathfrak{h}_{k}(\chi)
\end{aligned}
$$

where

$$
\begin{gathered}
g_{k}(\chi)=\frac{d}{d \chi} f_{k}(\chi)=\sum_{j=0}^{N} A_{j}^{k} \frac{d}{d \chi} \operatorname{cn}^{2 j}(\chi ; m)=\operatorname{scd}(\chi ; m) \sum_{j=0}^{N} a_{j}^{k} \operatorname{cn}^{2 j}(\chi ; m) \\
\mathfrak{h}_{k}(\chi)=\frac{d^{2}}{d \chi^{2}} f_{k}(\chi)=\sum_{j=0}^{N} A_{j}^{k} \frac{d^{2}}{d \chi^{2}} \operatorname{cn}^{2 j}(\chi ; m)=\sum_{j=0}^{N} \mathfrak{a}_{j}^{k} \operatorname{cn}^{2 j}(\chi ; m) \\
\text { in which }\left[a_{j}^{k}\right]=\left[A_{j}^{k}\right] \mathbb{G}, \quad \text { and }\left[\mathfrak{a}_{j}^{k}\right]=\left[A_{j}^{k}\right] \mathbb{F}
\end{gathered}
$$

$\mathbb{G}$ has dimensions $(2 N+1) \times(2 N+1)$ and $\mathbb{F}$ here has dimensions $(3 N+2) \times(3 N+2)$. The function $\operatorname{scd}(\chi)$ is

$$
\operatorname{scd}(\chi)=\operatorname{sn}(\chi) \operatorname{cn}(\chi) \operatorname{dn}(\chi)
$$

The Jacobian elliptic functions $\operatorname{sn}(\chi)$ and $\operatorname{dn}(\chi)$ appear only in this product combination in the Cnoidal Approximation theory. The transformation matrix $\mathbb{G}$ is also listed in Appendix A.

\section{The Cnoidal Approximation}

The unknown variables in a Cnoidal Approximation solution are $m, \alpha, U_{E}$ or $U_{S}, Q, R$, the $A_{j}$ coefficients for the stream function $\Psi(\chi, \zeta)$ and the $B_{j}$ coefficients for the water surface $\eta(\chi)$, for $j=0,1, \ldots N$, of which there are $2 N+7$.

The problem formulation provides $3 M+4$ implicit algebraic and nonlinear equations in these $2 N+7$ unknowns, each equation being cast in the form

$$
f_{i}\left(m_{1}, \alpha, U_{E} \text { or } U_{S}, Q_{1}, R_{1}, A_{j}, B_{j}\right)=0
$$


in which $m_{1}=1-m, Q_{1}=Q-\bar{U} h$ and $R_{1}=R-\frac{1}{2} C^{2}$. The equations are those identified in Section 2, and additionally the Laplace residual Eq. 21. They define the wave height

$$
f_{1}=\eta(X=0)-\eta(X=L / 2)-H
$$

the mean water level

$$
\begin{aligned}
f_{2} & =\frac{1}{L / 2} \int_{0}^{L / 2} \eta(X) d X \\
& =\frac{1}{\mathrm{~K}(m)} \int_{0}^{\mathrm{K}(m)} \eta(\chi) d \chi=\sum_{j} B_{j} \frac{1}{\mathrm{~K}(m)} \int_{0}^{\mathrm{K}(m)} \mathrm{cn}^{2 j}(\chi ; m) d \chi \\
& =\sum_{j} B_{j} \mathbf{I}_{j}
\end{aligned}
$$

the Eulerian current

$$
f_{3}=\frac{L}{T}-\bar{U}-U_{E}
$$

and the Stokes drift

$$
f_{4}=\frac{L}{T}-\frac{Q}{h}-U_{S}
$$

The kinematic free surface boundary condition (KFSBC) and the dynamic free surface boundary condition (DFSBC) are imposed along the free surface at $M$ discrete nodes over $0 \leq X \leq L / 2$, a half wave profile. These discrete points are distributed as

$$
X_{i}=\left(\frac{i-1}{M-1}\right)^{2} \frac{L}{2}, \quad \text { for } i=1,2, \ldots M
$$

to focus attention toward the crest where kinematic variations are most rapid for a cnoidal wave. The water surface elevation at $X_{i}$ is $\eta_{i}$ (Eq. 26).

The kinematic free surface boundary condition (KFSBC) at each of the $M$ free surface nodes is

$$
f_{2+3 i}=\Psi\left(X_{i}, \eta_{i}\right)+Q \quad \text { for } i=1,2, \ldots M
$$

and the dynamic free surface boundary condition (DFSBC) at each of the free surface nodes is

$$
f_{3+3 i}=\frac{1}{2}\left[U^{2}\left(X_{i}, \eta_{i}\right)+w^{2}\left(X_{i}, \eta_{i}\right)\right]+g \eta_{i}-R \quad \text { for } i=1,2, \ldots M
$$

Additionally, the free surface Laplace condition (Eq. 21) at the same $X_{i}$ free surface nodes is

$$
f_{4+3 i}=\left.\frac{\partial U}{\partial z}\right|_{\left(X_{i}, \eta_{i}\right)}-\left.\frac{\partial w}{\partial X}\right|_{\left(X_{i}, \eta_{i}\right)} \quad \text { for } i=1,2, \ldots M
$$

With $\partial U / \partial z-\partial w / \partial X$ scaled by the $(1+\zeta)$ multiplier from zero at the bed to a maximum at the free surface, Eqs. 44 will impose the Laplace equation to excellent precision throughout the solution field. 
In the above $f_{i}$ equations,

$$
L=\frac{2 \mathrm{~K}(m) h}{\alpha}
$$

and

$$
\begin{aligned}
\bar{U} & =-\frac{1}{L} \int_{0}^{L} U(X,-h) d X=-(g h)^{1 / 2} \frac{1}{\mathrm{~K}(m)} \int_{0}^{\mathrm{K}(m)} f(\chi) d \chi \\
& =-(g h)^{1 / 2} \sum_{j} A_{j} \frac{1}{\mathrm{~K}(m)} \int_{0}^{\mathrm{K}(m)} \mathrm{cn}^{2 j}(\chi ; m) d \chi \\
& =-(g h)^{1 / 2} \sum_{j} A_{j} \mathbf{I}_{j}
\end{aligned}
$$

The definite integrals

$$
\mathbf{I}_{j}=\frac{1}{\mathrm{~K}(m)} \int_{0}^{\mathrm{K}(m)} \mathrm{cn}^{2 j}(\chi ; m) d \chi
$$

follow analytically from the recurrence relationship

$$
\begin{gathered}
(2 j-1) m \mathbf{I}_{j}=-(2 j-2)(1-2 m) \mathbf{I}_{j-1}+(2 j-3)(1-m) \mathbf{I}_{j-2} \\
\text { where } \mathbf{I}_{0}=1, \mathbf{I}_{1}=\frac{e+m-1}{m} \text { and } e=\frac{\mathrm{E}(m)}{\mathrm{K}(m)}
\end{gathered}
$$

established from Gradshteyn \& Ryzhik (1965, §5.131(2)). $\mathrm{E}(m)$ is the Jacobian elliptic integral of the second kind with parameter $m$.

There are $3 M+4$ of implicit algebraic equations in $2 N+7$ unknowns. The problem can be uniquely specified, for $N=3,6,9, \ldots$ for which $M=3,5,7, \ldots$, but this does not provide a suitable algorithm. The choice of order $N$ is restrictive and the spatial resolution $M$ (through Eq. 41) of the free surface constraints is unlikely to be satisfactory.

The solution of a set of $3 M+4$ simultaneous implicit nonlinear algebraic equations in $2 N+7$ unknowns, where $M>2 N / 3+1$, is a familiar problem in numerical analysis in the context of nonlinear optimisation. A solution is established by seeking a minimum value for an objective function of the $2 N+7$ unknowns. The nonlinear optimisation algorithm adopted was the trust region method (Conn et al. 2000), based of Equations 37 through 43. The least squares objective function was

$$
\mathrm{O}\left(m_{1}, \alpha, U_{E} \text { or } U_{S}, Q_{1}, R_{1}, A_{j}, B_{j}\right)=f_{1}^{2}+\ldots+f_{4}^{2}+\left[f_{5}^{2}+\ldots+f_{3 M+4}^{2}\right] / M
$$

such that each of the seven distinct physical contributions to the problem formulations (Eqs. 37 through 40 and 42 through 44) is similarly weighted. Moderately significant overspecification, choosing $M$ principally to resolve the free surface boundary constraints, is physically advantageous. $M=20$ was routinely satisfactory.

To ensure the same prominence for the separate contributions to the Equation 48 objective function, it is numerically essential that each contribution has the same physical 
dimensions. This was achieved by non-dimensionalising every variable in the problem formulation by the shallow water length and time scales

$$
\mathbb{L}=h, \quad \mathbb{T}=(h / g)^{1 / 2}
$$

respectively.

A typical wave problem has 23 unknowns (at $N=8$, as subsequently adopted). Nonlinear optimisation in 23 dimensions may find mathematical solutions at local minima for the Equation 48 objective function. Identification of the unique physical solution requires some very careful attention to detail. Specific attention must be given to the initial solution estimate, to the convergence criterion, and to the predictions of field kinematics, especially acceleration. These issues are considered below.

Routine experience consistently confirms that the dominant issue is the relative fidelity of the initial solution estimate. Numerical optimisation in so many dimensions can rarely survive a poor initial estimate. And linear or Airy wave theory provides a poor initial estimate, except for infinitesimal waves. Three strategies were adopted, in sequential order.

(I) $m_{1}, \alpha, U_{E}$ or $U_{S}, Q_{1}, R_{1}$, and the coefficients $A_{j}, B_{j}$ through $A_{5}, B_{5}$ were assigned from an existing analytical theory to fifth order (Sobey 2012). In the analytical theory, there are " $i j$ " contributions at each order $\mathrm{i}$, the coefficients being designated $\mathrm{A}_{\mathrm{i} j}$ and $\mathrm{B}_{\mathrm{i} j}$ respectively. The $A_{j}$ and $B_{j}$ were assigned as

$$
A_{j}=\sum_{\mathrm{i}=0}^{5} \varepsilon^{\mathrm{i}} \mathrm{A}_{\mathrm{i} j}, \quad B_{j}=\sum_{\mathrm{i}=0}^{5} \varepsilon^{\mathrm{i}} \mathrm{B}_{\mathrm{i} j}
$$

respectively, where $\varepsilon=H / h$. The initial estimates for $A_{j}, B_{j}$ coefficients beyond $A_{5}, B_{5}$ were zero.

(II) For extreme waves, the analytical theory to fifth order is not reliable and strategy I generally fails. The analytical solution will not provide reasonable initial solution estimates for wave heights in excess of about $50 \%$ of the limit wave in shallow water, rising to about $75 \%$ in transitional water (Sobey 2012, Figure 8). For more extreme wave conditions, successful solutions were often achieved with initial solution estimates provided by prior solutions at gradually increasing wave height.

Assume a sequence of solutions $S(H)$, strictly $S(H ; h, T, \rho, M)$, where the parameters $h, T, \rho$ and $M$ are fixed. $S$ represents in turn elements of the solution vector $\left[m_{1}, \alpha, U_{E}\right.$ or $\left.U_{S}, Q_{1}, R_{1}, A_{j}, B_{j}\right]$. The strategy II initial solution estimate is

$$
S_{\text {Initial }}=S_{-1}
$$

where $S_{-1}$ is the solution vector at the next highest wave height $H_{-1}$ at which there was a successful solution. 
(III) Strategy II in turn also sometimes fails for the more extreme waves, especially in shallow water. The final strategy imposes a truncated Taylor series approximation. The strategy III initial solution estimate is

$$
S_{\text {Initial }}=S_{-1}+\frac{d S}{d H}\left(H-H_{-1}\right)
$$

where $d S / d H=\left(S_{-1}-S_{-2}\right) /\left(H_{-1}-H_{-2}\right)$. As before the "-1" subscript identifies the highest available successful solution. Similarly, "-2" identifies the next to highest successful solution.

Computations, at IEEE double precision, assigned convergence at a relative error in the non-dimensional objective function not exceeding $10^{-20}$, a choice suggested by preliminary numerical experiments. This is a very demanding convergence criterion, but the computational penalty was not excessive.

\section{Predicted Kinematics}

A complete list of predictive equations for the wave kinematics is included as Appendix B.

Figure 2 compares predictions of the key kinematics from the Sobey (2012) analytical cnoidal wave theory to fifth order (Cnoidal 5) with the present Cnoidal Approximation theory also at $N=5$ (Cnoidal Approximation 5 or CA 5) for a steady wave at depth $h=2 \mathrm{~m}$, period $T=10 \mathrm{~s}$, height $H=0.5 \mathrm{~m}$ and zero current, $U_{E}=0$. Part (a) shows the water surface elevation, part (b) the velocity components, and part (c) the acceleration components. The agreement is visually perfect, confirming the Cnoidal Approximation formulation and its coding implementation. The acceleration components are especially sensitive measures of predictive capability, as they involve double spatial differentiation of $\Psi$.

Figure 2 is a wave condition well within the validity range of the analytical theory (shown later in Figure 6); the dimensionless depth $\omega^{2} h / g$ is 0.080 and $H / H_{\text {Limit }}$ is 0.31 , where $H_{\text {Limit }}$ is the Williams (1985) prediction for the limit wave at the same dimensionless depth. There is nevertheless an important observation on the value of the Cnoidal Approximation theory, even at the same nominal order. Each theory involves compromises in the kinematic free surface boundary condition, the dynamic free surface boundary condition and the Laplacian residual. The extent of these compromises is explicitly included in the Eq. 48 objective function, or separately as the root-mean-square errors along the free surface:

$$
\begin{aligned}
\operatorname{rms}_{K} & =\frac{1}{L / 2} \int_{0}^{L / 2}[\Psi(X, \eta)+Q]^{2} d X \\
\operatorname{rms}_{D} & =\frac{1}{L / 2} \int_{0}^{L / 2}\left[\frac{1}{2}\left[U^{2}(X, \eta)+w^{2}(X, \eta)\right]+g \eta-R\right]^{2} d X \\
\operatorname{rms}_{L} & =\frac{1}{L / 2} \int_{0}^{L / 2}\left[\left.\frac{\partial U}{\partial z}\right|_{(X, \eta)}-\left.\frac{\partial w}{\partial X}\right|_{(X, \eta)}\right]^{2} d X
\end{aligned}
$$



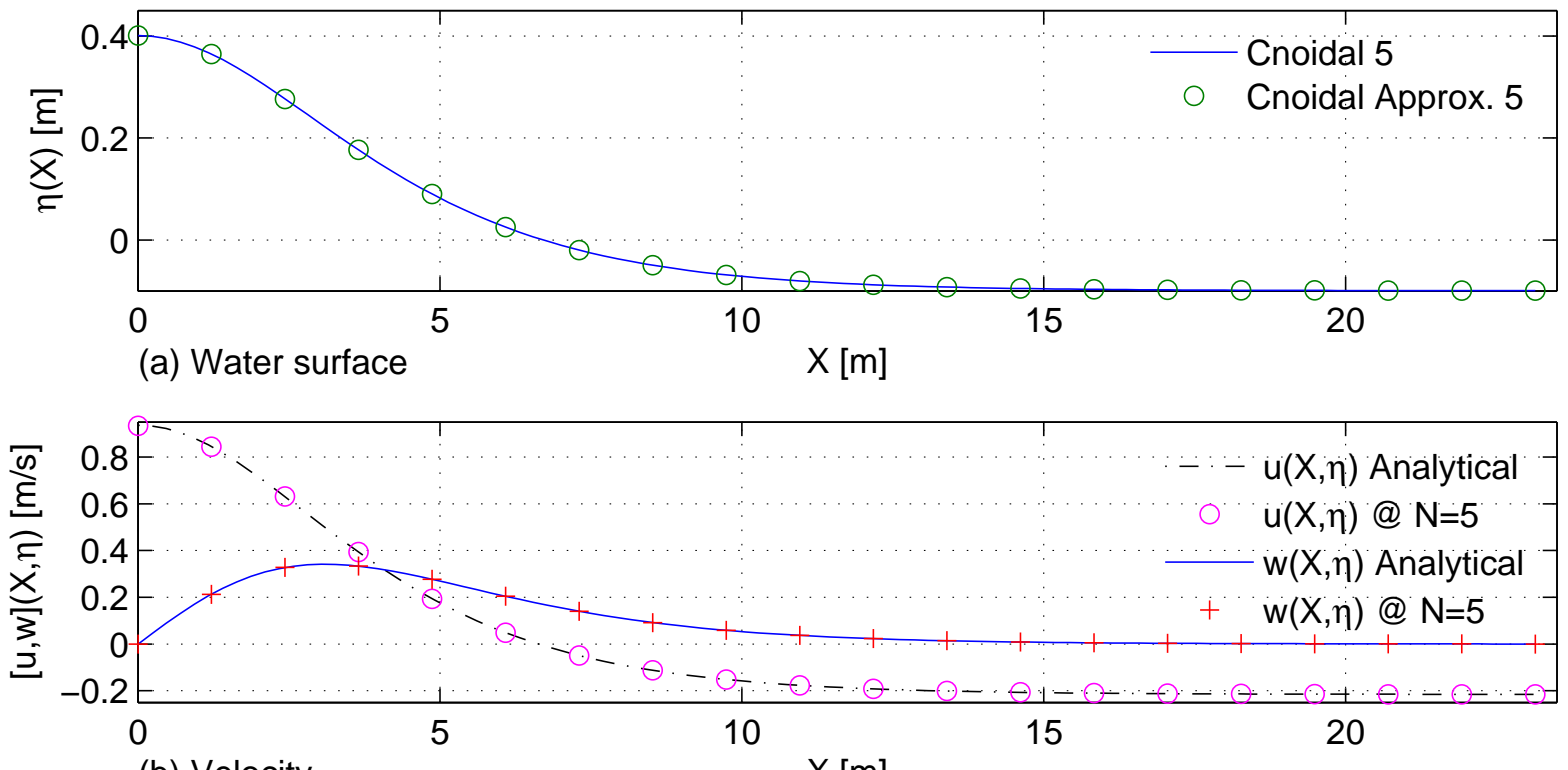

(b) Velocity

$X[\mathrm{~m}]$

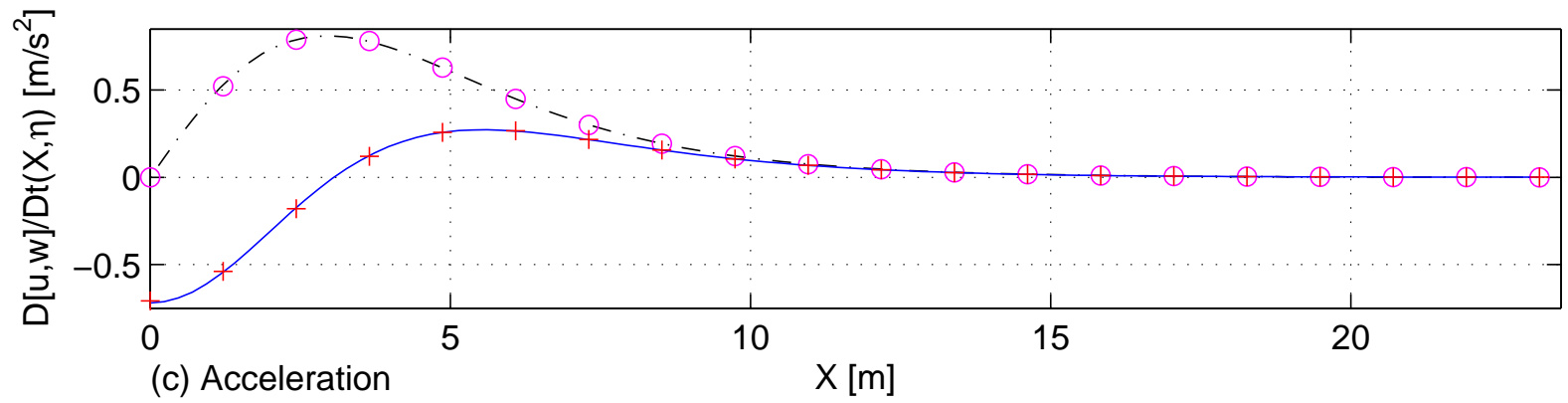

Figure 2: Analytical and Cnoidal Approximation Predictions at $N=5$ for $h=2 \mathrm{~m}, H=0.5$ $\mathrm{m}, T=10 \mathrm{~s}, U_{E}=0$.

\begin{tabular}{|l|llllll|}
\hline & Analytical & CA $N=5$ & CA 7 & CA 8 & CA 9 & CA 10 \\
\hline $\operatorname{rms}_{K}$ & $1.93 \mathrm{e}-5$ & $5.90 \mathrm{e}-7$ & $1.48 \mathrm{e}-8$ & $1.34 \mathrm{e}-8$ & $2.58 \mathrm{e}-8$ & $5.67 \mathrm{e}-7$ \\
$\operatorname{rms}_{D}$ & $1.01 \mathrm{e}-4$ & $3.80 \mathrm{e}-7$ & $1.29 \mathrm{e}-8$ & $6.26 \mathrm{e}-9$ & $3.60 \mathrm{e}-8$ & $3.67 \mathrm{e}-7$ \\
$\operatorname{rms}_{L}$ & 0 & $2.43 \mathrm{e}-7$ & $5.27 \mathrm{e}-9$ & $8.27 \mathrm{e}-10$ & $4.19 \mathrm{e}-9$ & $3.48 \mathrm{e}-8$ \\
\hline
\end{tabular}

Table 1: Dimensionless Root-Mean-Square Errors for $h=2 \mathrm{~m}, H=0.5 \mathrm{~m}, T=10 \mathrm{~s}, U_{E}=0$.

Numerical evaluation of the integrals adopted the trapezoidal rule, over 100 uniformly spaced points. In the analytical theory, the $f_{k}(\chi)$ are truncated strictly at fifth order and the Laplace residual is identically zero. Table 1 list the dimensionless rms errors for the wave conditions of Figure 2; scaling adopted the Eq. 49 length and time scales. 


\begin{tabular}{|l|llllll|}
\hline & Analytical & CA $N=5$ & CA 7 & CA 8 & CA 9 & CA 10 \\
\hline $\operatorname{rms}_{K}$ & $1.00 \mathrm{e}-2$ & $4.05 \mathrm{e}-4$ & $8.75 \mathrm{e}-5$ & $4.86 \mathrm{e}-5$ & $5.76 \mathrm{e}-5$ & $5.56 \mathrm{e}-5$ \\
$\operatorname{rms}_{D}$ & $2.35 \mathrm{e}-2$ & $3.77 \mathrm{e}-4$ & $8.13 \mathrm{e}-5$ & $4.69 \mathrm{e}-5$ & $5.50 \mathrm{e}-5$ & $9.19 \mathrm{e}-5$ \\
$\operatorname{rms}_{L}$ & 0 & $1.42 \mathrm{e}-4$ & $3.04 \mathrm{e}-5$ & $5.39 \mathrm{e}-5$ & $2.68 \mathrm{e}-5$ & $1.85 \mathrm{e}-4$ \\
\hline
\end{tabular}

Table 2: Dimensionless Root-Mean-Square Errors for $h=2 \mathrm{~m}, H=1.25 \mathrm{~m}, T=10 \mathrm{~s}, U_{E}=0$.

Also listed in this table are the rms errors for Cnoidal Approximation solutions at $N$ of 7 through 10. The numerical predictions at $N$ of 7 through 10 are visually identical with the $N=5$ solutions in Figure 2. The rms errors in Table 1 are all very small and confirm the predictive capability of the analytical theory and each of the CA results. The trends nevertheless provide a useful commentary on the respective theories. As expected, the rms errors for CA 5 are significantly smaller than for the fifth-order analytical theory. For the analytical theory, the error (by definition) is of order $\varepsilon^{6}=(H / h)^{6}=2.44 \mathrm{e}-4$; this is the order of magnitude for $\mathrm{rms}_{K}$ and $\mathrm{rms}_{D}$.

Error for the Cnoidal Approximation theory is somewhat different. In this case, order defines the truncation for $f(\chi)=f_{0}(\chi)$ in the stream function (Eqs. 15 and 25) and for the water surface elevation $\eta(X)$ (Eq. 26). Terms involving $\mathrm{cn}^{2 j}(\chi)$ for $j$ exceeding $N$ and up to $2 N+1$ appear in the $f_{k}(\chi)$, as outlined in the discussion following Eq. 27. The CA rms errors are impacted by the convergence criterion for the nonlinear optimisation only when the convergence criterion are not adequate; stable results were routinely achieved at the adopted relative error of $10^{-20}$ in the objective function.

The trend in the $\mathrm{CA}$ results in Table 1 provides an excellent overview on the value of CA order. There is initially some apparent advantage in increasing the order, but this quickly reaches saturation. Further increases are of marginal value, and increasing rms errors become apparent. These increases are routinely associated with decreasingly robust solution convergence. Extensive numerical experience has suggested $N=8$ as an optimal choice for CA order. This $N$ is deceptively small. $\Psi, U$ and $\partial U / \partial z$ actually include terms to $\mathrm{cn}^{2(2 N+1)}, w, \partial U / \partial X$ and $\partial w / \partial z$ terms to $\mathrm{cn}^{2(2 N)}$ and $\partial w / \partial X$ terms to $\mathrm{cn}^{2(3 N+2)}$.

Figure 3 is a wave condition $\left(h=2 \mathrm{~m}, H=1.25 \mathrm{~m}, T=10 \mathrm{~s}, U_{E}=0 ; \omega^{2} h / g=0.080\right.$, $H / H_{\text {Limit }}=0.79$ ) well outside the validity range of the analytical theory (see Figure 6 ). As expected, the analytical predictions for velocity and especially acceleration are not credible. The Cnoidal Approximation predictions at $N$ of 5 and 7 through 10 were again visually identical. The CA 8 predictions are credible. They seem physically plausible and the rms errors, listed in Table 2, are suitably small. Note that the rms errors are rather larger for this nearer limit wave than was the case for the Figure 2 wave, but the errors remain respectably small.

The hybrid analytical-numerical Stokes/Fourier Approximation (FA) is well established, as well as the almost default extension of this FA theory into the shallow water domain at higher truncation orders. Higher orders may indeed push the apparent application of the 

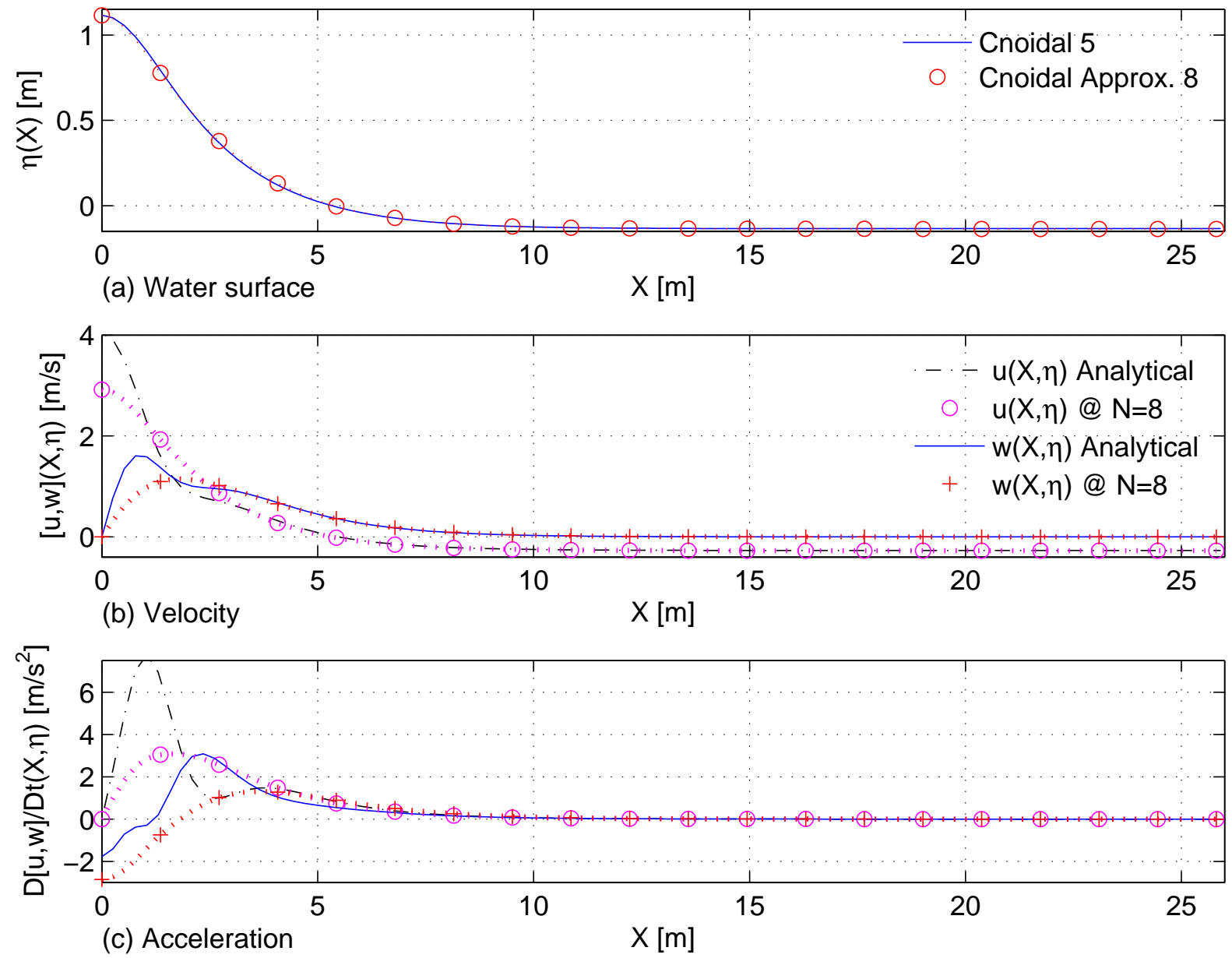

Figure 3: Analytical and Cnoidal Approximation Predictions at $N=8$ for $h=2 \mathrm{~m}, H=1.25$ $\mathrm{m}, T=10 \mathrm{~s}, U_{E}=0$.

FA theory into shallow water but it remains a Stokes approximation with optimal credibility in deeper water. Difficulties become most apparent in the extended flat trough of shallow water waves, where oscillations begin to appear in water surface predictions, rather more in velocity predictions and especially in acceleration predictions. Figure 4, for the Figure 3 wave conditions, shows these oscillations for the water surface profile predictions. Included are the CA 8 prediction from Figure 3, together with Fourier Approximation predictions (code from Sobey 1989) at $N=10$ and $N=15$. At the scale of part (a), the oscillations are not especially visible in the $\eta(X)$ predictions, though they can be detected in the FA acceleration predictions. The FA oscillations are clear in part (b) at expanded scale. The CA 8 prediction, even at expanded scale, remains well behaved and credible. The complementary roles of the present hybrid analytical-numerical Cnoidal Approximation (CA) in shallow water and the hybrid analytical-numerical Fourier Approximation (FA) in deep water was anticipated above. The temptation to push the CA theory to deeper water 


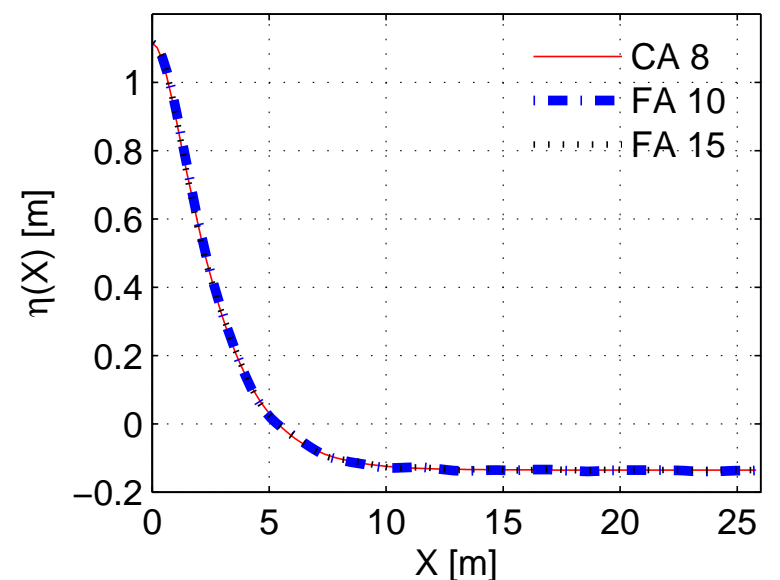

(a) $\eta(X)$ predictions

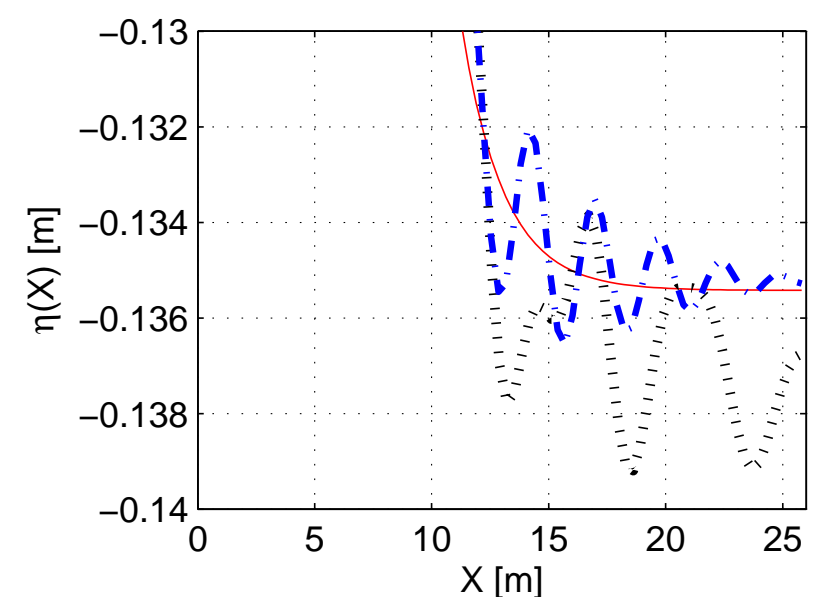

(b) $\eta(X)$ at expanded vertical scale

Figure 4: Cnoidal and Fourier Approximation Predictions for $h=2 \mathrm{~m}, H=1.25 \mathrm{~m}, T=10 \mathrm{~s}$, $U_{E}=0$.

\begin{tabular}{|l|llllll|}
\hline & Analytical & CA $N=5$ & CA 7 & CA 8 & CA 9 & CA 10 \\
\hline $\operatorname{rms}_{K}$ & $3.93 \mathrm{e}-3$ & $7.99 \mathrm{e}-4$ & $2.23 \mathrm{e}-4$ & $1.30 \mathrm{e}-4$ & $7.63 \mathrm{e}-5$ & $4.77 \mathrm{e}-5$ \\
$\operatorname{rms}_{D}$ & $5.78 \mathrm{e}-3$ & $7.90 \mathrm{e}-4$ & $2.40 \mathrm{e}-4$ & $1.40 \mathrm{e}-4$ & $8.17 \mathrm{e}-5$ & $5.13 \mathrm{e}-5$ \\
$\operatorname{rms}_{L}$ & 0 & $2.12 \mathrm{e}-4$ & $5.61 \mathrm{e}-5$ & $4.48 \mathrm{e}-5$ & $2.95 \mathrm{e}-5$ & $1.48 \mathrm{e}-5$ \\
\hline
\end{tabular}

Table 3: Dimensionless Root-Mean-Square Errors for $h=20 \mathrm{~m}, H=11.0 \mathrm{~m}, T=10 \mathrm{~s}, U_{E}=0$.

is fortunately constrained by rapidly declining sensitivity to the Jacobian parameter $m$ as $m$ becomes very small.

Figure 5 is a wave condition $\left(h=20 \mathrm{~m}, H=11.0 \mathrm{~m}, T=10 \mathrm{~s}, U_{E}=0 ; \omega^{2} h / g=0.80\right.$, $\left.H / H_{\text {Limit }}=0.85\right)$ in transitional water and beyond the validity range of the analytical theory (see Figure 6). That the analytical predictions for accelerations lack credibility requires close inspection of the acceleration traces. $D u / D t$ along the water surface oscillates twice below and then above the smoothly varying CA 8 prediction. Similarly $D w / D t$ starts well above the CA 8 trace at the crest and then oscillates below and then back above. $D w / D t$ at the crest is subsequently shown to be perhaps the key indicator of the predictive capability of both the analytical and the CA theories.

Again also, the Cnoidal Approximation predictions at $N$ of 5 and 7 through 10 were visually identical. The associated rms errors are listed in Table 3. Note that the rms errors are again rather larger than was the case for the Figure 2 wave. This was expected as the Figure 5 wave is in much deeper water, stretching the credibility of the shallow water response implicit in the stream function representation (Eqs. 15 through 18). The wave height is also relatively extreme. Nevertheless, the errors remain respectably small. 


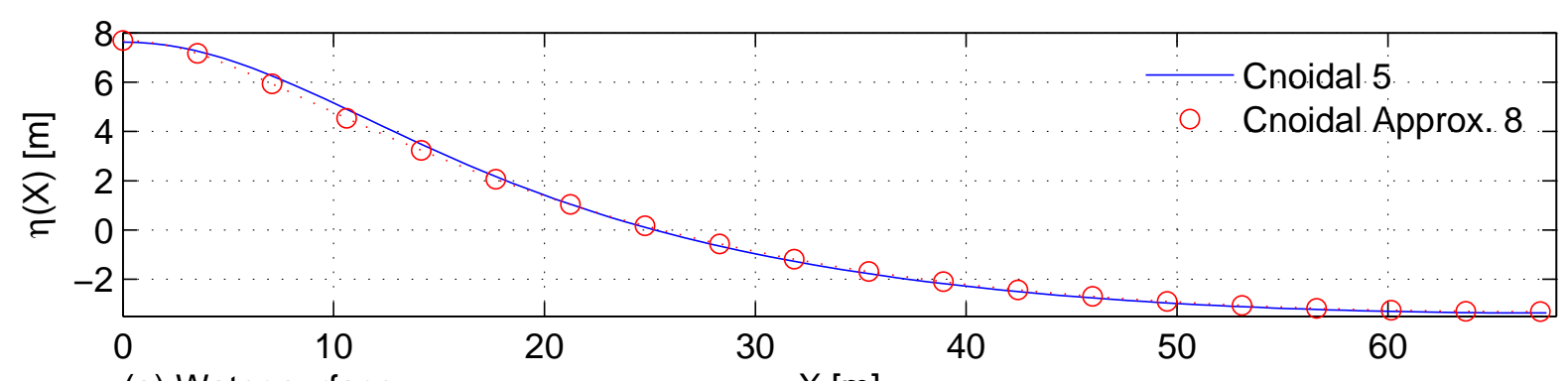

(a) Water surface

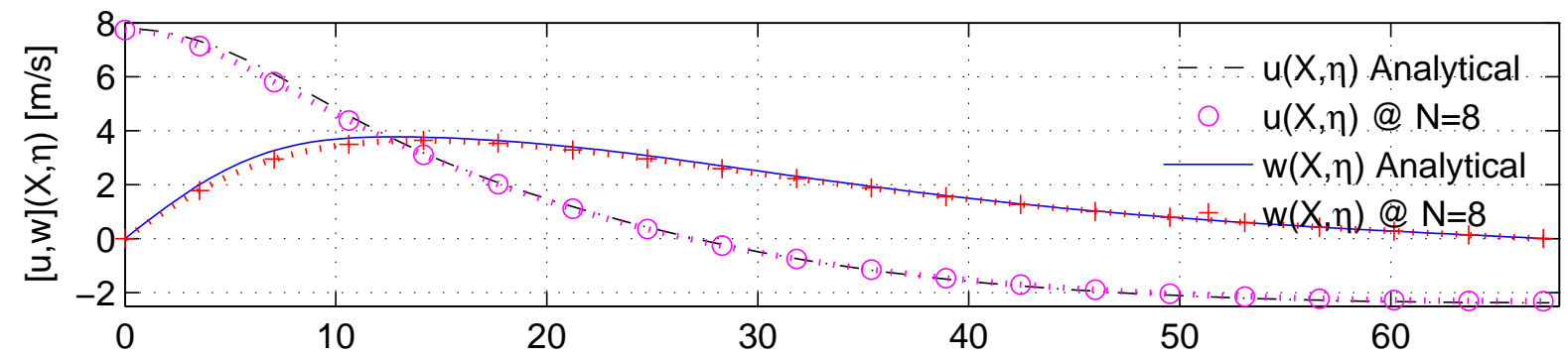

(b) Velocity

$X[\mathrm{~m}]$

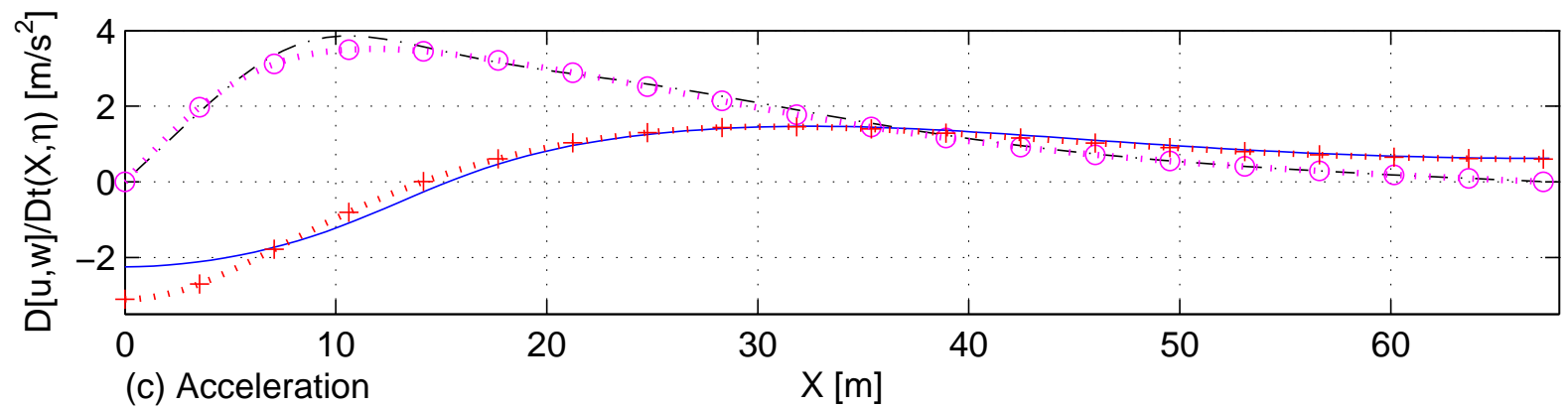

Figure 5: Analytical and Cnoidal Approximation Predictions at $N=8$ for $h=20 \mathrm{~m}, H=11.0$ $\mathrm{m}, T=10 \mathrm{~s}, U_{E}=0$.

The relationship in magnitudes between the analytical errors and the various CA errors is maintained.

\section{$7 \quad$ Limits of Validity}

The limits of validity of the CA theory does not extend to the entire spectrum of length scales (water depth $h$, wave height $H$ and wave length $L$ ). As previously outlined (Equation 6 and discussion between Equations 26 and 27), solitary waves $(L=\infty$, and $m=1)$ violate the CA problem formulation. In contrast, analytical theories for cnoidal waves may provide viable predictions at $m=1$. Both analytical theories for cnoidal waves and the present CA theory experience difficulties on approach to the limit wave.

It is apparent from Figures 3 and 5 that the CA theory can provide credible wave predictions for significantly higher waves than was possible with the fifth order analytical 


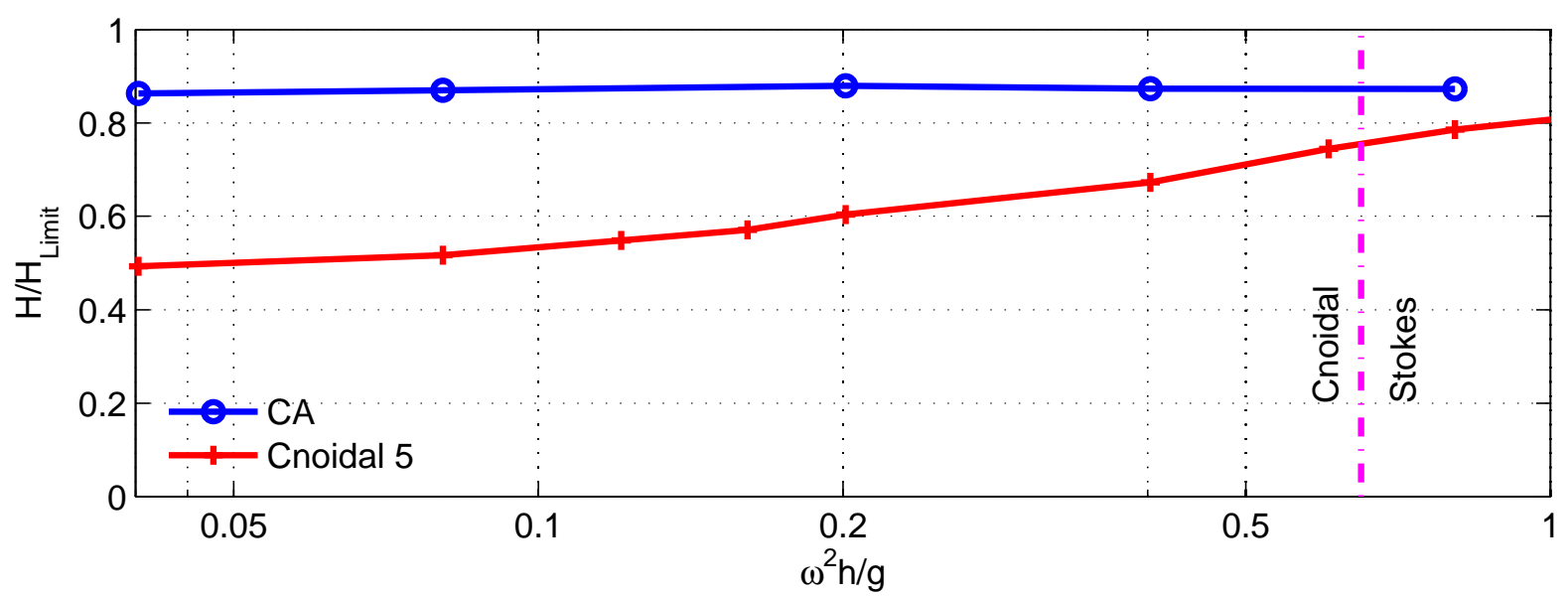

Figure 6: Comparative validity of analytical Cnoidal 5 theory and numerical CA theory.

theory. The established (see Sobey 2012, Figure 8) limits of validity for the analytical theory are reproduced in Figure 6 , focussing on the range $\omega^{2} h / g \lesssim 0.65$ there suggested as appropriate for cnoidal wave theory. (Stokes-style wave theory is appropriate in deeper water.) The analytical theory seems to provide reasonable predictions up to wave heights increasing from about 0.5 of the limit wave in very shallow water $\left(\omega^{2} h / g \rightarrow 0\right)$ to marginally above 0.7 of the limit wave height in transition water $\left(\omega^{2} h / g \rightarrow 0.65\right)$.

The CA approach to the limit wave has been explored through a data base of solutions at depths $h$ ranging from 1 to $50 \mathrm{~m}$ at period $T=10 \mathrm{~s}$. Attention was specifically directed to the evolution with increasing wave height of the

(a) Dimensionless wave length $L / h$

A moderate increase in the domain aspect ratio $L / h$ above the Airy (linear) wave theory prediction of

$$
\frac{L_{\text {Airy }}}{h}=\frac{2 \pi}{k h}, \quad \text { where } \quad \omega^{2}=g k \tanh k h, k=2 \pi / L_{\text {Airy }}
$$

is expected as the wave height is increased.

(b) Dimensionless crest elevation $\eta_{\mathrm{Cr}} / H$

$\eta_{\mathrm{Cr}}=\eta(0,0)$ is expected to increase above $H / 2$ as $H$ increases. At the limit wave, the crest is a stagnation point in the steady frame, so that $\eta_{\mathrm{Cr}}$ increases toward $R / g$ (Eq. 2), which approaches closer to $H$ as the depth decreases.

(c) Dimensionless horizontal crest velocity $u_{\mathrm{Cr}} / C$

$u_{\mathrm{Cr}}=u(0,0)$ is expected to increase toward the phase speed $C$ at the limit wave. This is a further consequence of the steady-frame stagnation point at the crest for the limit wave. 
(d) Dimensionless vertical crest acceleration $(D w / D t)_{\mathrm{Cr}} / g$

$(D w / D t)_{\mathrm{Cr}}(D w / D t$ at $X=0, t=0)$ is expected to decrease toward $-g / 2$ at the limit wave (Longuet-Higgins \& Fox 1977).

In each case, the progression with increasing $H$ is expected to be smooth. Any departure from a smooth progression will identify a failure of the CA theory.

But analytical continuity may result in smooth transitions to flawed predictions. Comparison with the existing fifth-order analytical theory will provide confirmation for small to moderately extreme wave heights, as suggested in Figure 6. Complementary support for moderately extreme to limit waves has been extracted from the Williams (1985) tables. Direct use of these tabulated predictions requires interpolation (in $h / g T^{2}$ and $H / g T^{2}$ ) over a two-dimensional and irregular grid of tabulated predictions. Bi-cubic interpolation over the irregular grid was adopted, and some scatter in the predictions is expected. The Williams (1985) predictions for the limit wave seem secure; interpolated values are used in the present definition of $H_{\text {Limit }}$. But the transition from moderately extreme to the limit wave are rather less secure, being characterised (Williams 1985, p. 16) as "less than satisfactory". Of solution characteristics (a) through (d) listed above, predictions of $L / h$ only are listed in this moderately extreme to the limit wave region.

It is appropriate to note also that the Williams tables provide essentially a Stokes approximation, extended to very high order, "about 20 for deep-water waves and to 80 for shallow-water waves" (Williams 1985, p.16). It is not certain that the very high order in shallow water has not been impacted by limited machine precision. There is no discussion of this possibility. There is nonetheless evidence (minor profile oscillations) of declining applicability in shallow water in some of the interpolated predictions at depths approaching $1 \mathrm{~m}$.

Figure 7 summarises the evolution for $h=2 \mathrm{~m}$ at $N=5$. (Figure 2 is a sample at $H=0.5$ $\mathrm{m}$ from this sequence of solutions). Figure 7 parts (a) through (d) correspond to the listed items (a) through (d) identified for specific attention. The solid dots are the numerical CA 5 predictions, the dashed line is the analytical cnoidal 5 predictions and the solid line is the predictions interpolated from the Williams (1985) tables. In each part figure, the wave height scale has been truncated at the limit wave, which is listed in the figure caption. The $L / h$ predictions, part (a), correspond closely up to $H \approx 1.15 \mathrm{~m}$, and the CA 5 and Cnoidal 5 predictions up to the limit wave. The $\eta_{\mathrm{Cr}} / H$ predictions, part (b), show good agreement throughout. The CA 5 and Williams-interpolated predictions for $u_{\mathrm{Cr}} / C$, part (c), show good agreement up to $H \approx 1.25 \mathrm{~m}$. Beyond this height, the $\mathrm{CA} 5$ predictions remain smooth, as expected from analytical continuity. The Williams-interpolated predictions have a sudden trend change to $u_{\mathrm{Cr}} / C=1$ at the limit wave. There is clearly a rapid change expected in this region. The upper limit is secure and the lower limit is credible. As reported above, Williams table predictions in this region are not especially secure and only integral properties are listed. Without resolution of this rapid change, interpolated predictions become the sudden linear trends seen clearly in parts (c) and (d). The prediction uncertainty is clearly marked.

Regardless, the CA 5 prediction at the limit wave, $u_{\mathrm{Cr}} / C \approx 0.75$, is not acceptable. The 


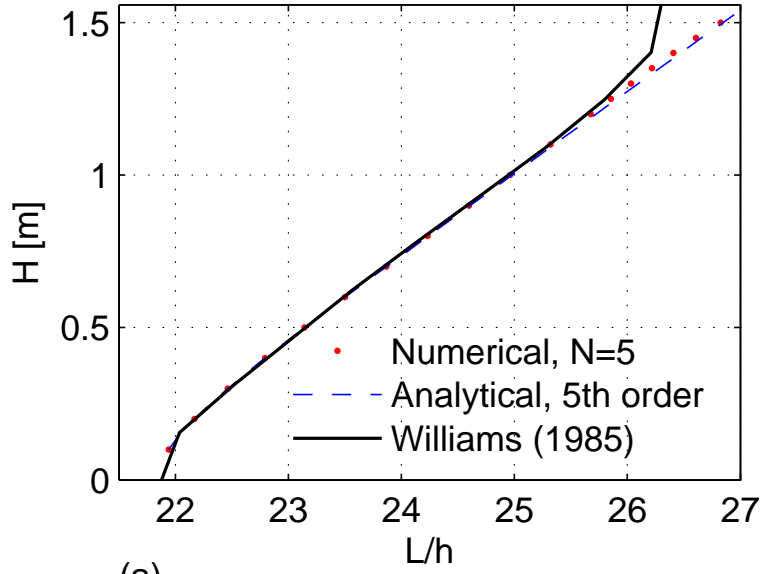

(a)

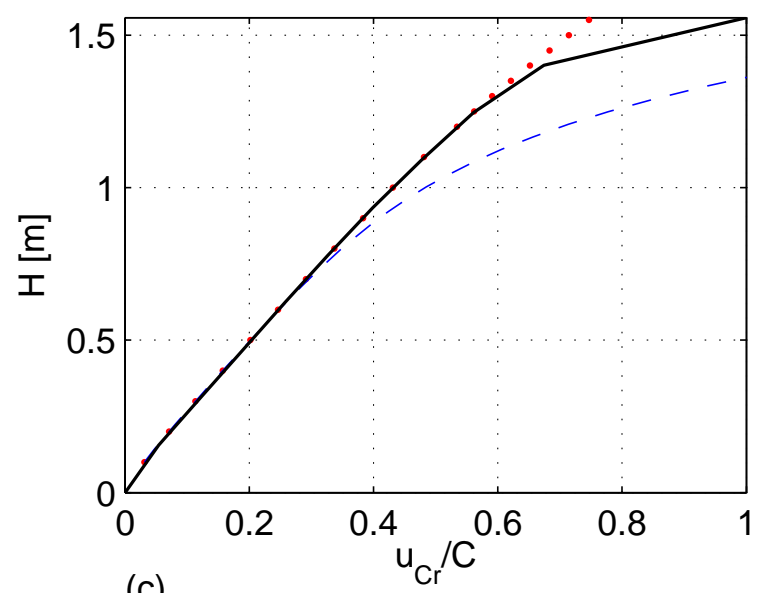

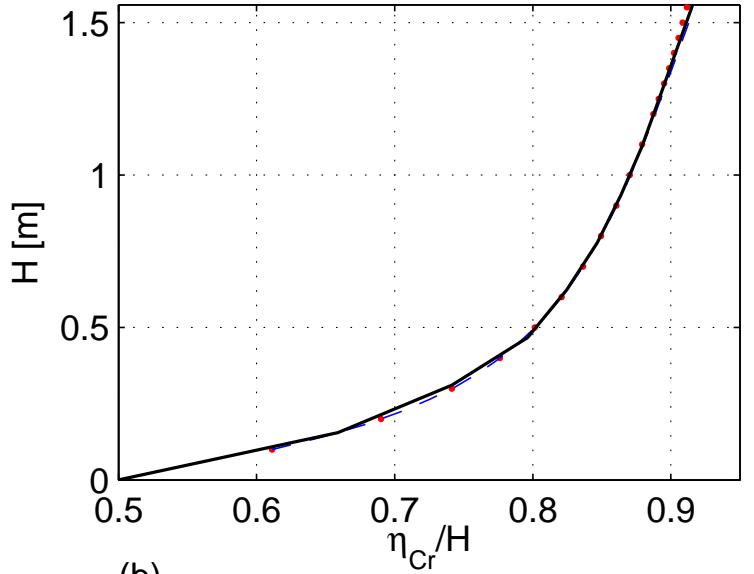

(b)

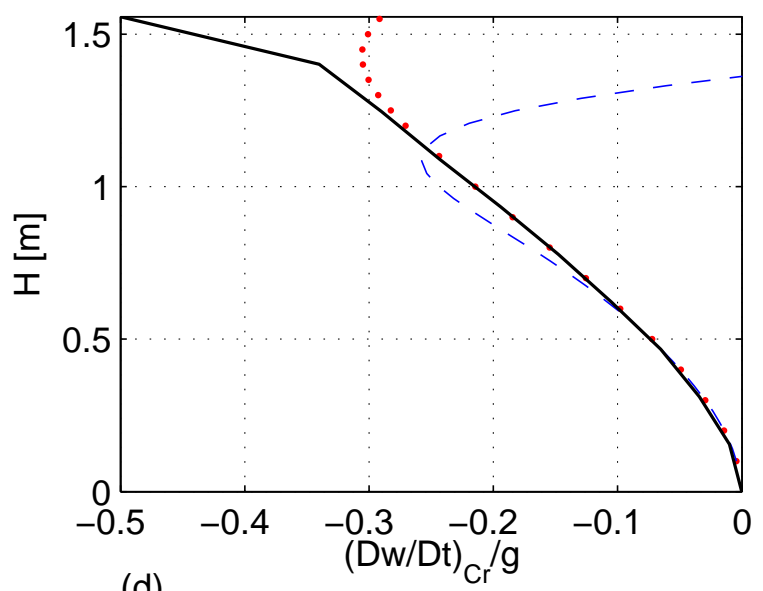

(d)

Figure 7: Solution Evolution with Wave Height at $N=5$ for $h=2 \mathrm{~m}, T=10 \mathrm{~s}, U_{E}=0$. $H_{\text {Limit }}=1.59 \mathrm{~m}$

analytical Cnoidal 5 predictions separate at only moderate wave height, reaching $u_{\mathrm{Cr}} / C=$ 1 at $H \approx 1.35 \mathrm{~m}$, well below the limit wave. These are unacceptable predictions, an observation strongly emphasised by part (d) where the analytical predictions are low at moderate wave heights and experience a rapid trend reversal toward zero vertical acceleration at $H \approx 1.35 \mathrm{~m}$. This rapid trend reversal in the analytical predictions for $(D w / D t)_{\mathrm{Cr}}$ is the clearest indication that the analytical theory has been extended beyond its region of applicability. The CA 5 and Williams-interpolated predictions closely correspond to $H \approx$ $1.15 \mathrm{~m}$, beyond which the CA 5 predictions for $(D w / D t)_{\mathrm{Cr}}$ experience a trend reversal similar to the analytical predictions. Once again, this trend reversal identifies the limit of applicability for the CA 5 predictions at $h=2 \mathrm{~m}$.

The CA 8 predictions at the same depth, Figure 8, are very much more satisfactory. The response patterns in parts (a) through (d) are similar to the CA 5 predictions in Figure 7, except that the CA 8 predictions follow the Williams-interpolated predictions 


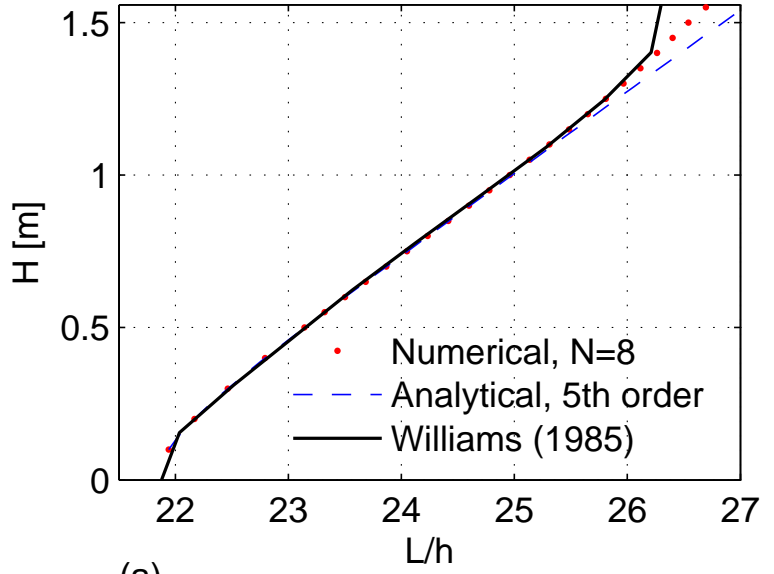

(a)

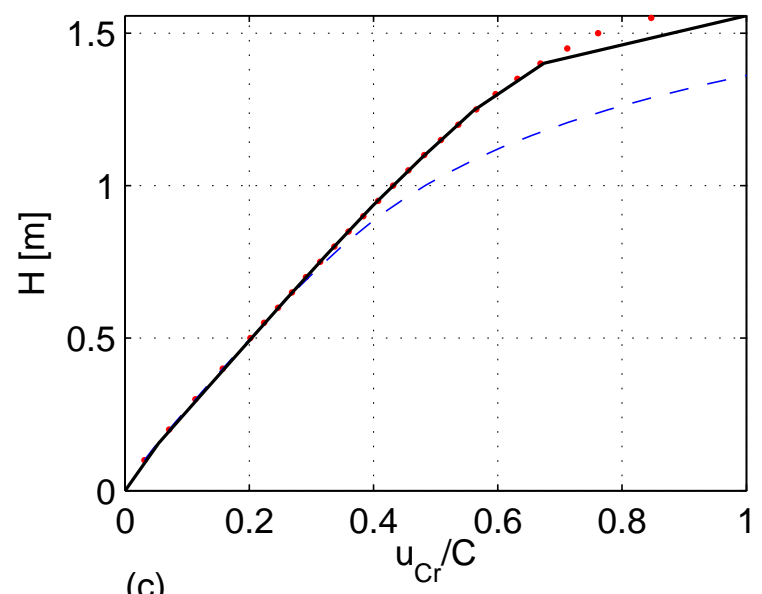

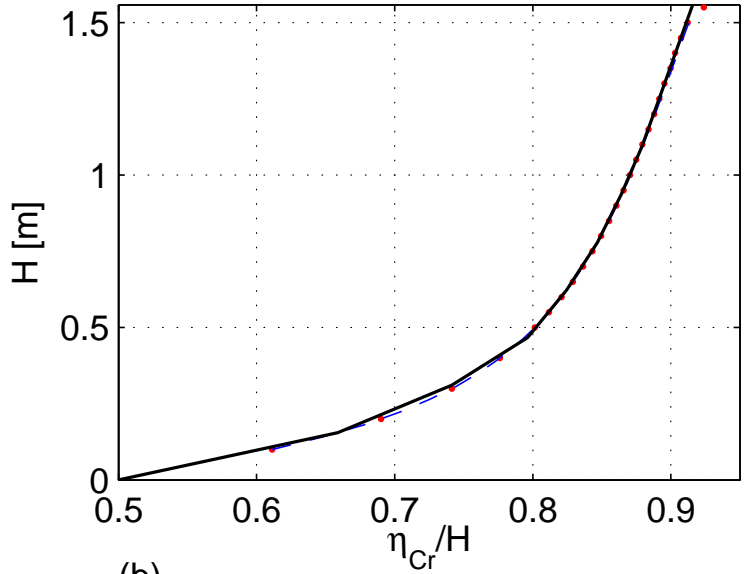

(b)

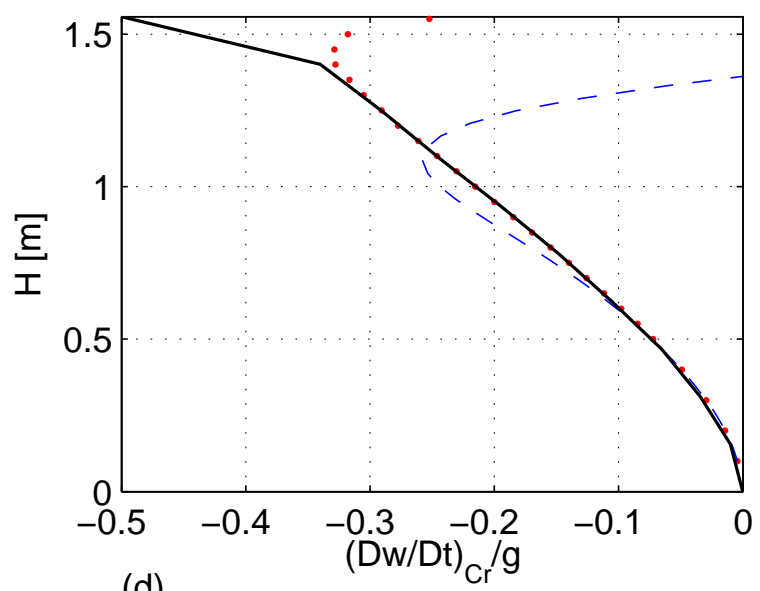

(d)

Figure 8: Solution Evolution with Wave Height at $N=8$ for $h=2 \mathrm{~m}, T=10 \mathrm{~s}, U_{E}=0$. $H_{\text {Limit }}=1.59 \mathrm{~m}, H_{\text {Valid }}=1.38 \mathrm{~m}$.

now beyond $H \approx 1.35 \mathrm{~m}$. Further increases in the truncation order $N$ do not significantly impact this response. This was anticipated in the discussion related to Tables 1 through 3 , to the extent that order $N=8$ has been routinely adopted.

It is clear from Figure 8(c) and (d) that the present CA formulation can not reach the limit wave. A smooth crest profile (Eq. 26) is assumed, which conflicts with the discontinuous crest with included angle $2 \pi / 3$ expected at the limit wave. The upper limit of validity of the CA theory can be identified by the separation of the CA and Williams-interpolated predictions for $u_{\mathrm{Cr}}$ and $(D w / D t)_{\mathrm{Cr}}$. The separation is amplified for $(D w / D t)_{\mathrm{Cr}}$. The upper limit of validity is identified as the wave height

$$
H_{\text {Valid }} \quad \text { where }\left.\frac{D w}{D t}\right|_{\mathrm{Cr}} ^{\mathrm{CA} 8}-\left.\frac{D w}{D t}\right|_{\mathrm{Cr}} ^{\text {Williams }}=0.005 \mathrm{~g}
$$

$H_{\text {Valid }}$ is listed in the Figure 8 caption. 


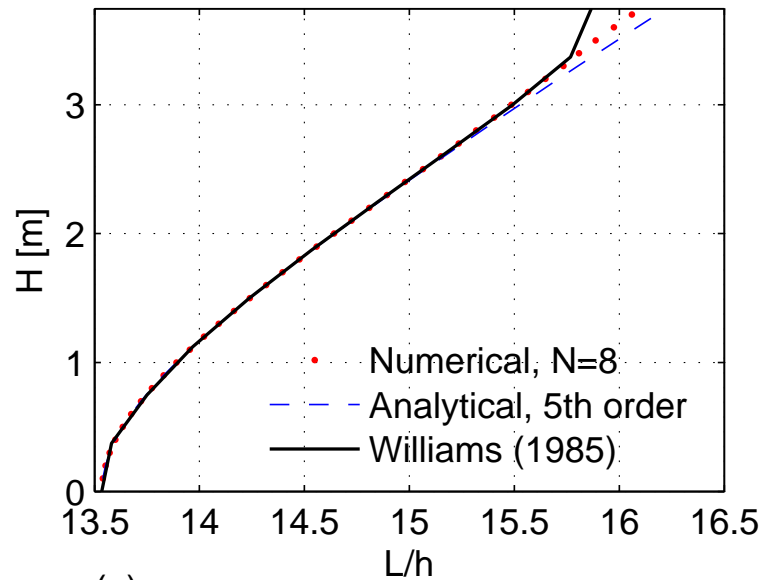

(a)

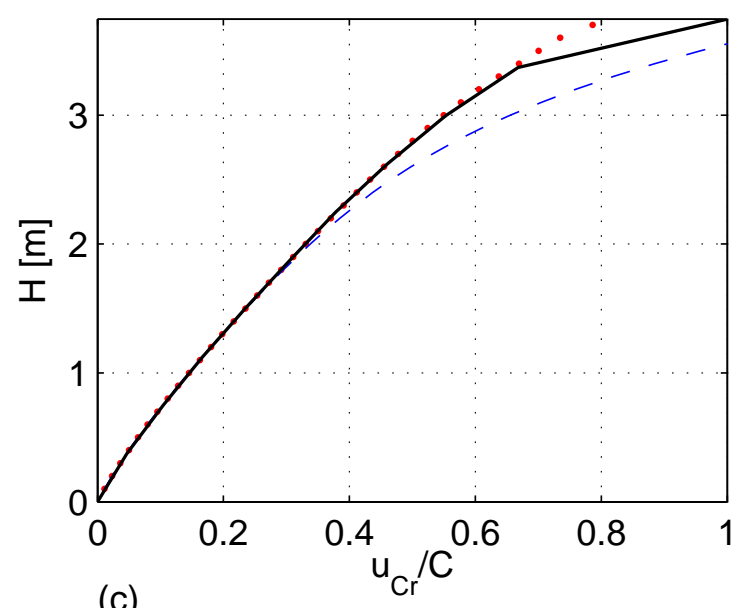

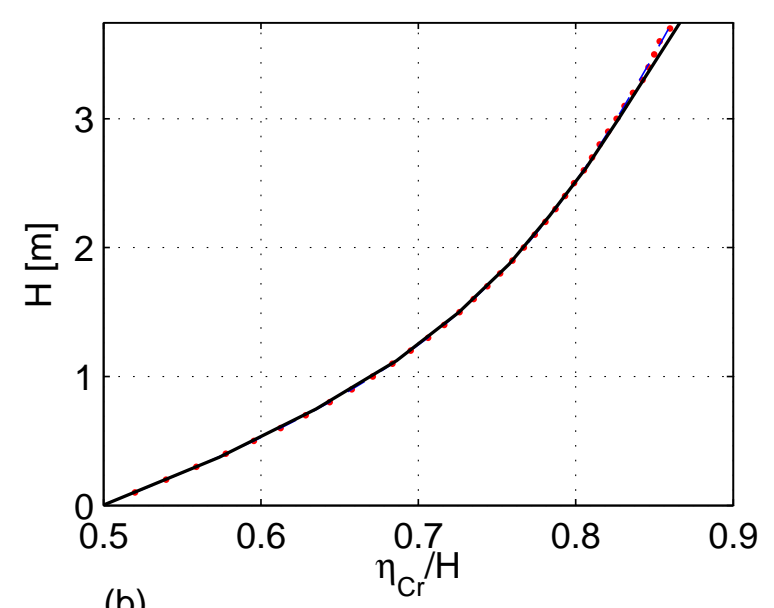

(b)

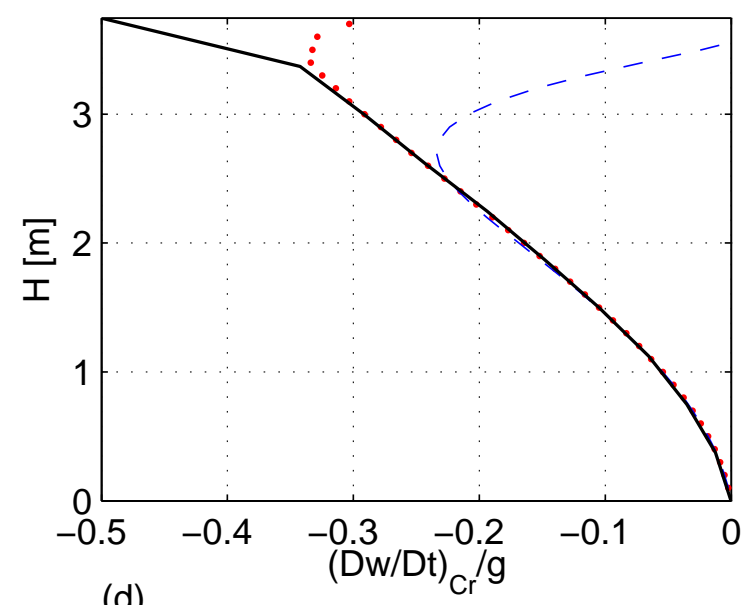

(d)

Figure 9: Solution Evolution with Wave Height at $N=8$ for $h=5 \mathrm{~m}, T=10 \mathrm{~s}, U_{E}=0$. $H_{\text {Limit }}=3.78 \mathrm{~m}, H_{\text {Valid }}=3.32 \mathrm{~m}$.

Almost identical response patterns are repeated as the depth is increased, for example in Figures 9 and 10 at depths of 5 and $20 \mathrm{~m}$ respectively.

Estimated values of $H_{\text {Valid }}$, following the Eq. 55 definition, at depths from 1 to $20 \mathrm{~m}$ are included in Figure 6 . CA 8 predictions, where appropriate $\left(\omega^{2} h / g \lesssim 0.65\right)$, seem to be routinely valid up to about $88 \%$ of the limit wave height. The CA 8 theory extends to significantly higher wave heights than the analytical theory. There is no expectation that the present CA theory can extend closer to the limit wave.

\section{Conclusions}

A Cnoidal Approximation (CA) wave theory for steady progressive waves in shallow water is described and explored. The formulation of the theory is analytical, building on existing 


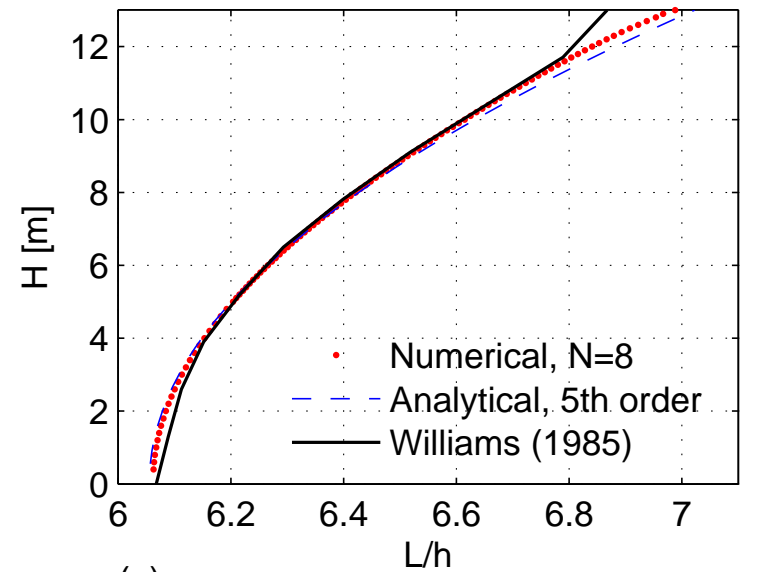

(a)

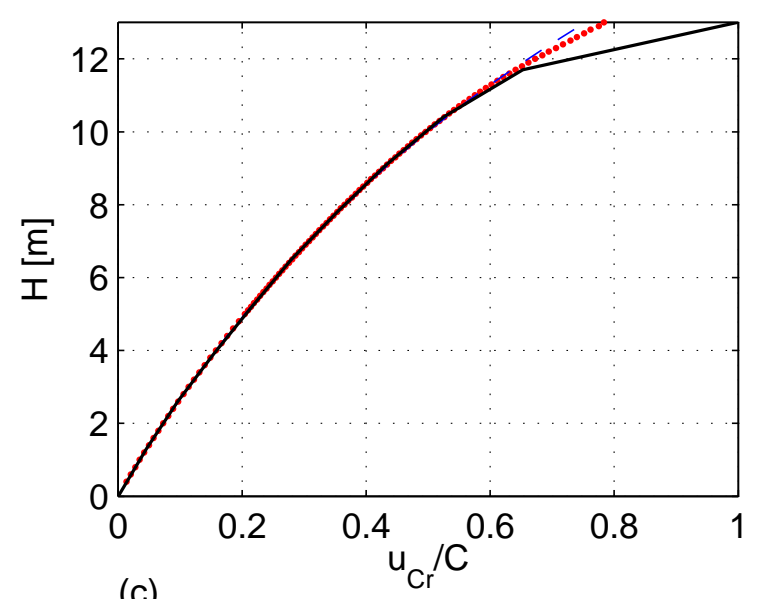

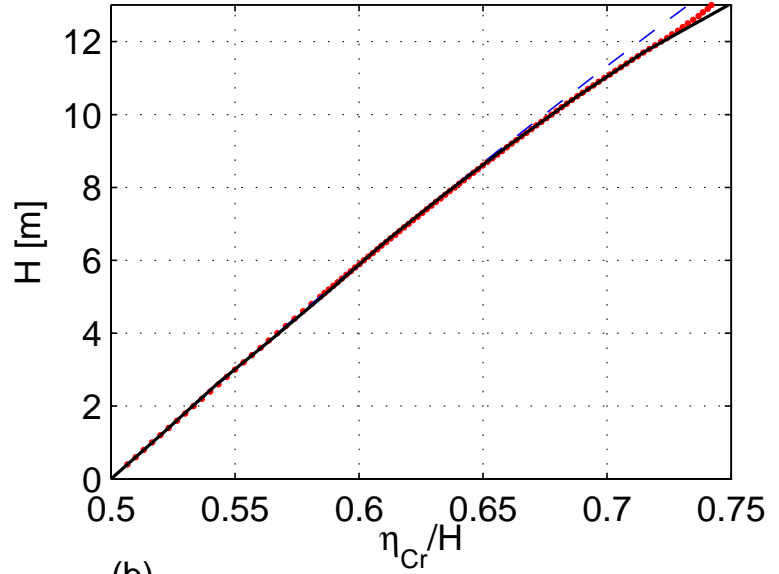

(b)

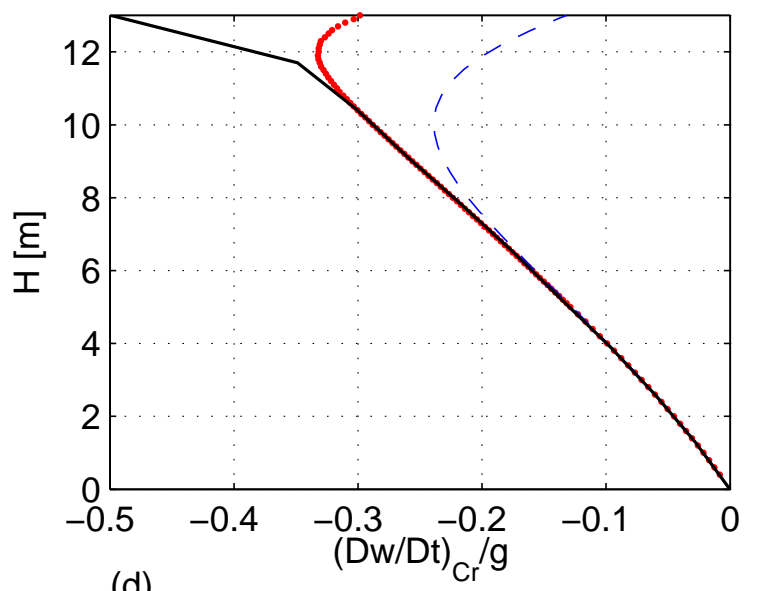

(d)

Figure 10: Solution Evolution with Wave Height at $N=8$ for $h=20 \mathrm{~m}, T=10 \mathrm{~s}, U_{E}=0$. $H_{\text {Limit }}=12.98 \mathrm{~m}, H_{\text {Valid }}=11.33 \mathrm{~m}$.

analytical theories for cnoidal waves. The solution is numerical, organised as a problem in nonlinear optimisation.

The analytical formulation is based on the Lamb (1932) series solution for the Laplace equation, which has horizontal and vertical scaling suitable for shallow water waves. The adopted solution for the stream function $\Psi$ exactly satisfies the bottom boundary condition and the periodic lateral boundary condition. Much of the balance of the analytical formulation is classical, the kinematic free surface boundary condition, the dynamic free surface boundary condition, the definition of wave height, the mean water level constraint and the dispersion relationship. Imposition of the periodic lateral boundary condition specifically excludes solitary wave solutions.

Truncation of the stream function series at order $N$ introduces a small residual error in the field equation. This residual error is zero at the bottom and largest in magnitude at the free surface. It is accommodated through a free surface Laplace condition, along with 
the traditional kinematic and dynamic free surface boundary conditions.

The analytical formulation is completed by series expansions in $\mathrm{cn}^{2 j}(\chi)$ for $j=0,1,2, \ldots N$ for the function $f(\chi)$ in the stream function and for the water surface elevation $\eta(\chi)$. The stream function, velocity components and velocity gradients require first and second $\chi$ derivatives of $\mathrm{cn}^{2 j}(\chi)$, which are provided analytically by the definition of suitable transformation matrices.

The balance of the solution is completed numerically. The problem formulation provides $3 M+4$ implicit and nonlinear algebraic equations in $2 N+7$ unknowns. The numerical solution is accomplished as a problem in nonlinear optimisation, based on the Eq. 48 least squares objective function. A trust region algorithm was adopted. Numerical solution issues such as variable scaling, initial solution estimation and convergence criteria are outlined in the text.

The balance of the kinematics are available from predictive equations summarised in Appendix B.

Confirmation of the CA-predicted kinematics is provided by comparison (Figure 2, Table 1) with the analytical Cnoidal 5 theory, but only within the range of validity of the analytical theory.

Errors in the CA theory have been characterised by the root-mean-square errors in the kinematic free surface boundary condition, the dynamic free surface boundary condition and the Laplace residual respectively. In all cases, the rms errors are very small. The optimum appears to be CA 8 . Beyond $N=8$, there seems to be some increase in the rms error levels, presumably a consequence of the impact of finite machine precision on the convergence criteria in the nonlinear optimisation algorithm. The rms error levels at CA 8 are very acceptable, in comparison with solution unknowns that have been scaled to order one.

Two further wave comparisons (Figures 3, Table 2 in shallow water and Figures 5, Table 3 in transitional water), show moderately extreme waves beyond the validity range of the analytical Cnoidal 5 theory. In both cases, the CA 8 predictions seem appropriate. There is subsequent confirmation in Figures 8 and 10.

Exploration of the CA theory concludes with an investigation of the limits of validity of the CA predictions. The predictions are compared (Figures 7 through 10) with the analytical Cnoidal 5 theory and also predictions interpolated from the Williams tables, an extremely high order Stokes approximation. As expected, satisfactory response is demonstrated by the Cnoidal 5 theory only for small to moderate wave heights. The CA predictions extend the validity to higher waves, and the benefit of increasing the truncation order is clear. The upper wave hight limit of validity of the CA 8 theory is identified at significant departure of the CA 8 predictions from the William-interpolated predictions. This $H_{\text {Valid }}$ can not reach the limit wave $H_{\text {Limit }}$, as the discontinuous crest of the limit wave is precluded by the CA theory.

The estimates for $H_{\text {Valid }}$ are consolidated on Figure 6 . The CA theory remains valid to about $88 \%$ of the limit wave height, throughout the shallow to transitional range $\left(\omega^{2} h / g \lesssim\right.$ 0.65) of applicability. 


\section{A Cnoidal Transformation Matrices}

(1) $d^{2} \mathrm{cn}^{2 j}(\chi) / d \chi^{2}$

Analytical differentiation leads to powers and products of $\operatorname{Jacobian} \operatorname{sn}(\chi), \operatorname{cn}(\chi)$ and $\operatorname{dn}(\chi)$ terms. These powers and products are transformed to sums of powers of $\mathrm{cn}^{2}(\chi)$, using identities among these functions based on $\operatorname{sn}^{2}(\chi)=1-\operatorname{cn}^{2}(\chi)$ and $\operatorname{dn}^{2}(\chi)=1-m+m \mathrm{cn}^{2}(\chi)$ [Gradshteyn \& Ryzhik $(1965, \S 8.154(4,5))$ ]. The generic result is

$$
\begin{aligned}
\frac{d^{2}}{d \chi^{2}} \mathrm{cn}^{2 j}(\chi)=2 j(2 j-1)(1-m) \mathrm{cn}^{2 j-2}(\chi)+4 j^{2}(2 m-1) & \mathrm{cn}^{2 j}(\chi) \\
- & 2 j(2 j+1) m \mathrm{cn}^{2 j+2}(\chi)
\end{aligned}
$$

The upper left corner of the equivalent transformation matrix is

$$
\mathbb{F}=\left[\begin{array}{cccccc}
0 & 0 & 0 & 0 & 0 & \ldots \\
2-2 m & 8 m-4 & -6 m & 0 & 0 & \ldots \\
0 & 12-12 m & 32 m-16 & -20 m & 0 & \ldots \\
0 & 0 & 30-30 m & 72 m-36 & -42 m & \ldots \\
0 & 0 & 0 & 56-56 m & 128 m-64 & -72 m \\
\vdots & \vdots & \vdots & \vdots & \vdots & \vdots
\end{array}\right]
$$

(2) $d \operatorname{cn}^{2 j}(\chi) / d \chi$

In a similar manner,

$$
\frac{d}{d \chi} \operatorname{cn}^{2 j}(\chi)=-2 j \operatorname{scd}(\chi) \operatorname{cn}^{2 j-2}(\chi)
$$

The equivalent transformation matrix is

$$
\mathbb{G}=\left[\begin{array}{cccccc}
0 & 0 & 0 & 0 & 0 & \ldots \\
-2 & 0 & 0 & 0 & 0 & \ldots \\
0 & -4 & 0 & 0 & 0 & \ldots \\
0 & 0 & -6 & 0 & 0 & \ldots \\
0 & 0 & 0 & -8 & 0 & \ldots \\
\vdots & \vdots & \vdots & \vdots & \vdots & \vdots
\end{array}\right]
$$




\section{B Complete Kinematics}

Given a successful Cnoidal Approximation solution, the complete kinematics are available as:

$$
\begin{array}{rlrl}
\Psi(X, z) & =\sum_{k=0}^{N}(-1)^{k} \frac{(1+\zeta)^{2 k+1}}{(2 k+1) !} f_{k}(\chi) & \eta(X) & =\sum_{j=0}^{N} B_{j} \mathrm{cn}^{2 j}(\chi ; m) \\
U(X, z) & =\frac{1}{h} \sum_{k=0}^{N}(-1)^{k} \frac{(1+\zeta)^{2 k}}{(2 k) !} f_{k}(\chi) & w(X, z) & =-\frac{\alpha}{h} \sum_{k=0}^{N}(-1)^{k} \frac{(1+\zeta)^{2 k+1}}{(2 k+1) !} g_{k}(\chi) \\
\left.\frac{\partial U}{\partial X}\right|_{(X, z)} & =\frac{\alpha}{h^{2}} \sum_{k=0}^{N}(-1)^{k} \frac{(1+\zeta)^{2 k}}{(2 k) !} g_{k}(\chi) & \left.\frac{\partial U}{\partial z}\right|_{(X, z)} & =\frac{1}{h^{2}} \sum_{k=1}^{N}(-1)^{k} \frac{(1+\zeta)^{2 k-1}}{(2 k-1) !} f_{k}(\chi) \\
\left.\frac{\partial w}{\partial X}\right|_{(X, z)} & =-\frac{\alpha^{2}}{h^{2}} \sum_{k=0}^{N}(-1)^{k} \frac{(1+\zeta)^{2 k+1}}{(2 k+1) !} \mathfrak{h}_{k}(\chi) & \left.\frac{\partial w}{\partial z}\right|_{(X, z)}=-\left.\frac{\partial U}{\partial X}\right|_{(X, z)} \\
\left.\frac{D u}{D t}\right|_{(X, z)} & =U \frac{\partial U}{\partial X}+w \frac{\partial U}{\partial z} & \left.\frac{D w}{D t}\right|_{(X, z)} & =U \frac{\partial w}{\partial X}+w \frac{\partial w}{\partial z} \\
p_{d}(X, z)=\rho R-\frac{1}{2} \rho\left(U^{2}+w^{2}\right)
\end{array}
$$

\section{References}

Conn, A. R., Gould, N. I. M. \& Toint, P. L. (2000), Trust-Region Methods, MPS/SIAM Series on Optimization, SIAM Society for Industrial \& Applied Mathematics, Philadelphia, PA.

Engevik, L. (2003), 'A comment on the series solution of the solitary-wave problem', Zeitschrift für angewandte Mathematik und Physik 54, 179-183.

Fenton, J. D. (1979), 'A high-order cnoidal wave theory', Journal of Fluid Mechanics 94, 129-161.

Gradshteyn, I. S. \& Ryzhik, I. M. (1965), Table of Integrals, Series and Products, Academic Press, New York.

Keller, J. B. (1948), 'The solitary wave and periodic waves in shallow water', Communications on Pure and Applied Mathematics 1, 323-339.

Laitone, E. V. (1960), 'The second approximation to cnoidal and solitary waves', Journal of Fluid Mechanics 9, 430-444. 
Lamb, H. (1932), Hydrodynamics, 6 edn, Cambridge University Press, Cambridge.

Longuet-Higgins, M. S. \& Fox, M. J. H. (1977), 'Theory of the almost-highest wave: the inner solution', Journal of Fluid Mechanics 80, 721-741.

Sobey, R. J. (1989), 'Variations on Fourier wave theory', International Journal for Numerical Methods in Fluids 9, 1453-1467.

Sobey, R. J. (2012), 'Steep progressive waves in deep and shallow water', Engineering and Computational Mechanics. In Press; accepted May 2010.

Williams, J. M. (1985), Tables of Progressive Gravity Waves, Pitman, Boston. 